\title{
ON COMMUTATIVE POWER-ASSOCIATIVE ALGEBRAS OF DEGREE TWO $\left.{ }^{(}\right)$
}

BY

\section{A. A. ALBERT}

1. Introduction. The study of the structure of any simple commutative power-associative algebra $\mathfrak{A}$ over a field $\mathfrak{F}$ begins with the case where $\mathfrak{F}$ is algebraically closed. We shall also assume that the characteristic of $\mathfrak{F}$ is prime to 30 . Then $\mathfrak{A}$ has a unity quantity 1 which is expressible as the sum $1=u_{1}+\cdots+u_{t}$ of pairwise orthogonal (absolutely) primitive idempotents $u_{i}$. The integer $t$ is unique and is called the degree of $\mathfrak{A}$. It is known that if $t>2$, the algebra $\mathfrak{A}$ is a classical Jordan algebra $\left({ }^{2}\right)$. The structure of algebras of degree two is not known and will be considered here.

Every simple algebra $\mathfrak{A}$ of degree two contains an idempotent $u \neq 1$ and there is a corresponding decomposition

$$
\mathfrak{A}=\mathfrak{U}_{u}(1)+\mathfrak{A}_{u}(1 / 2)+\mathfrak{U}_{u}(0),
$$

where $\mathfrak{A}_{u}(\alpha)$ consists of all quantities $x_{\alpha}$ of $\mathfrak{A}$ such that $u x_{\alpha}=\alpha x_{\alpha}$. It is known( $\left.{ }^{3}\right)$ that $\mathfrak{A}_{u}(1)$ and $\mathfrak{A}_{u}(0)$ are orthogonal subalgebras of $\mathfrak{A}$, that $\left[\mathfrak{A}_{u}(1 / 2)\right]^{2}$ $\subseteq \mathfrak{A}_{u}(1)+\mathfrak{A}_{u}(0)$, and that

$$
\mathfrak{A}_{u}(\lambda) \mathfrak{A}_{u}(1 / 2) \subseteq \mathfrak{A}_{u}(1 / 2)+\mathfrak{A}_{u}(1-\lambda) \quad(\lambda=0,1) .
$$

We say that $\mathfrak{A}$ is $u$-stable (stable relative to the idempotent $u$ ) if the stronger condition

$$
\mathfrak{A}_{u}(\lambda) \mathfrak{A}_{u}(1 / 2) \subseteq \mathfrak{A}_{u}(1 / 2) \quad(\lambda=0,1)
$$

holds. Moreover, we call an algebra $\mathfrak{A}$ a stable algebra if $\mathfrak{A}$ is $u$-stable for every idempotent $u$ of $\mathfrak{A}$.

The main result of this paper will be stated as follows.

THEOREM 1. Let $\mathfrak{A}$ be a simple commutative power-associative algebra of degree $t>1$ over its center $\mathfrak{F}$ whose characteristic is prime to 30 . Then $\mathfrak{A}$ is stable if and only if $\mathfrak{U}$ is a Jordan algebra.

Mr. Louis Kokoris has constructed $\left({ }^{4}\right)$ a class of $u$-stable central simple

Presented to the Society, April 26, 1952, under the title New power-associative algebras and their derivations; received by the editors May 12, 1952.

(1) This paper was sponsored in part by the Office of Naval Research.

(2) See Theorem 8 of the author's $A$ theory of power-associative commutative algebras, Trans. Amer. Math. Soc. vol. 69 (1950) pp. 503-527. We shall refer to this paper as CPA.

(3). These results were given in Power-associative rings, Trans. Amer. Math. Soc. vol. 62 (1948) pp. 552-593.

(4) These results will be published in the Proc. Nat. Acad. Sci. U. S. A. in 1952. 
commutative power-associative algebras $\subseteq$ of degree two and dimension $4 p$ over a field $\mathfrak{F}$ of characteristic $p$. These algebras are not Jordan algebras since $\mathfrak{S}_{u}(1)$ has a $(p-1)$-dimensional radical, while $\mathfrak{A}_{u}(1)$ is simple for every simple Jordan algebra $\mathfrak{A}$.

Each of the algebras of Kokoris contains a simple subalgebra $\mathfrak{T}$ of dimension $3 p$ which is constructed in terms of the algebra $\mathscr{B}$ of all polynomials in $x$ with coefficients in $\mathfrak{F}$ such that $x^{p}=0$. We shall obtain the derivation algebra $\mathfrak{D}$ of $\mathfrak{S}$ and shall show that it coincides with the derivation algebra of $\mathfrak{T}$. Moreover, $\mathfrak{D}$ is the direct sum of the three-dimensional central simple Lie algebra of two-rowed matrices of trace zero and the well known $p$-dimensional simple Lie algebra of Witt, that is, the derivation algebra of $\mathfrak{B}$.

We close our study with an extensive generalization of the algebras $\mathfrak{S}$ and $\mathfrak{T}$ of Kokoris. We generalize $\mathfrak{B}$ to be the algebra $\mathfrak{B}_{r}$ of all polynomials in $x_{1}, \cdots, x_{r}$ with $x_{i}^{p}=0$, and define a certain set $\&$ of derivations $D_{i j}=-D_{j i}$ for all integers $i \geqq 0$ and $j \geqq 0$. Let $\mathfrak{D}_{m}$ be the subset of $\mathbb{E}$ consisting of all $D_{i j}$ with $i \leqq m$ and $j \leqq m$, and define a corresponding commutative algebra $\mathfrak{T}\left(\mathfrak{F}_{r}, \mathfrak{D}_{m}\right)=\mathfrak{B}_{r}+y_{0} \mathfrak{B}_{r}+\cdots+y_{m} \mathfrak{B}_{r}$ of dimension $p^{r}(m+2)$ over $\mathfrak{F}$ of characteristic $p$, for every $m>0$ where $\left(y_{i} b\right) c=y_{i}(b c),\left(y_{i} b\right)\left(y_{j} c\right)=\left(b D_{i j}\right) c-b\left(c D_{i j}\right)$ for every $b$ and $c$ of $\mathscr{B}_{r}$ and $i, j=0, \cdots, m$. This algebra can be imbedded in a $u$-stable algebra $\subseteq\left(\mathfrak{B}_{r}, \mathfrak{D}_{m}\right)=\mathfrak{T}\left(\mathfrak{B}_{r}, \mathfrak{D}_{m}\right)+u \mathfrak{F}_{r}$, and the algebras $\subseteq$ and $\mathfrak{T}$ of Kokoris are the case $r=m=1$ of the algebras $\mathfrak{S}\left(\mathfrak{B}_{r}, \mathfrak{D}_{m}\right)$ and $\mathfrak{T}\left(\mathfrak{B}_{r}, \mathfrak{D}_{r}\right)$. The new algebras are central simple power-associative algebras and contain other central simple algebras whose dimensions need not even be divisible by $p$.

2. Structure properties of $u$-stable algebras. If $\mathfrak{A}$ is a simple commutative power-associative algebra over $\mathfrak{F}$ the scalar multiples $\alpha 1$ of the unity quantity 1 of $\mathfrak{A}$ form a subalgebra of $\mathfrak{A}$, and the mapping $\alpha \leftrightarrow \alpha 1$ is an isomorphism of this subalgebra and $\mathfrak{F}$. We shall identify this subalgebra with $\mathfrak{F}$, shall write $\alpha$ rather than $\alpha 1$, and shall regard $\mathfrak{F}$ as being a subset (actually a subring) of $\mathfrak{A}$.

We shall assume that $\mathfrak{F}$ is an algebraically closed field of characteristic prime to 30 and that $\mathfrak{A}$ has degree two. Then $1=u+v$ for an idempotent $u \neq 1$, so that $u$ and $v$ are orthogonal idempotents. Since $u$ and $v$ are absolutely primitive, we have

$$
\mathfrak{A}_{u}(1)=u \mathfrak{F}+\mathfrak{R}_{1}, \quad \mathfrak{A}_{u}(0)=\mathfrak{A}_{v}(1)=v \mathfrak{F}+\mathfrak{N}_{0},
$$

where $\mathfrak{N}_{\lambda}$ is a nilalgebra and is the radical of $\mathfrak{A}_{u}(\lambda)$. Define

$$
\mathfrak{C}=\mathfrak{A}_{u}(1) \oplus \mathfrak{A}_{u}(0)=u \mathfrak{F}+v \mathfrak{F}+\mathfrak{N}^{\prime},
$$

where $\mathfrak{N}^{\prime}=\mathfrak{N}_{1} \oplus \mathfrak{R}_{0}$ is then the radical of $\mathfrak{E}$. The idempotents $u$ and $v$ are the unity quantities of $\mathfrak{A}_{u}(1)$ and $\mathfrak{A}_{u}(0)$ respectively, and we have

$$
(a b) u=(a u) b=a(b u)=(a u)(b u), \quad(a b) v=(a v) b=a(v b)=(a v)(b v),
$$

for every $a$ and $b$ of $\mathfrak{E}$. 
The following known $\left({ }^{5}\right)$ result is a basic tool in our investigation.

Lemma 1. Let $x$ and $y$ be in $\mathfrak{A}_{u}(1 / 2)$. Then

$$
x y=\alpha+z,
$$

where $\alpha$ is in $\mathfrak{F}$ and $z$ is in $\mathfrak{N}^{\prime}$.

A commutative algebra $\mathfrak{A}$ over a field $\mathfrak{F}$ of characteristic prime to 30 is power-associative if and only if

$$
\begin{aligned}
& 4[(w x)(y z)+(w y)(x z)+(w z)(x y)] \\
&= w[x(y z)+y(z x)+z(x y)]+x[y(z w)+z(w y)+w(y z)] \\
&+y[z(w x)+w(x z)+x(z w)]+z[w(x y)+x(y w)+y(w x)]
\end{aligned}
$$

for every $w, x, y, z$ of $\mathfrak{A}$. This identity is of obvious importance in our analysis.

If $x$ is in $\mathfrak{A}_{u}(1 / 2)$, we apply Lemma 1 to see that

$$
x^{2}=\alpha+z,
$$

where $z$ is nilpotent and $\alpha$ is in $\mathfrak{F}$. If $\alpha=0$, then $x$ is nilpotent. Otherwise the algebra $\mathfrak{F}[x]$ contains 1 and $x^{-1}$ and we shall call $x$ nonsingular.

Lemma 2. If $\mathfrak{A}$ is simple and u-stable, there is a nonsingular quantity in $\mathfrak{A}_{u}(1 / 2)$.

For $\mathfrak{C}$ is not simple and $\mathfrak{A}_{u}(1 / 2) \neq 0$. If $x^{2}$ were in $\mathfrak{N}^{\prime}$ for every $x$ of $\mathfrak{A}_{u}(1 / 2)$, then $2 x y=(x+y)^{2}-x^{2}-y^{2}$ would be in $\mathfrak{R}^{\prime}$ for every $x$ and $y$ of $\mathfrak{A}_{u}(1 / 2)$, that is, $\left[\mathfrak{H}_{u}(1 / 2)\right]^{2} \subseteq \mathfrak{N}^{\prime}$. Also $\mathfrak{S A}_{u}(1 / 2) \subseteq \mathfrak{A}_{u}(1 / 2)$ since $\mathfrak{A}$ is $u$-stable. Define $\mathfrak{M}=\mathfrak{N}^{\prime}+\mathfrak{A}_{u}(1 / 2)$ and see that $\mathfrak{M A}=\mathfrak{N}^{\prime} \mathfrak{C}+\mathfrak{C} \mathfrak{A}_{u}(1 / 2)+\mathfrak{N}^{\prime} \mathfrak{A}_{u}(1 / 2)+\left[\mathfrak{A}_{u}(1 / 2)\right]^{2}$ $\subseteq \mathfrak{N}^{\prime}+\mathfrak{A}_{u}(1 / 2)+\mathfrak{A}_{u}(1 / 2)+\mathfrak{R}^{\prime} \subseteq \mathfrak{M}$, that is, $\mathfrak{M}$ is an ideal of $\mathfrak{A}$. This is impossible since $\mathfrak{A}$ is simple and $\mathfrak{A} \not \neq \mathfrak{M} \geqq \mathfrak{A}_{u}(1 / 2) \neq 0$.

The result of Lemma 2 implies the following result.

Lemma 3. Let $x$ be a nonsingular element of $\mathfrak{A}_{u}(1 / 2)$ where $\mathfrak{A}$ is $u$-stable. Then there exists a quantity $c$ in $\mathfrak{F}\left[x^{2}\right] \subseteq \mathfrak{C}$ such that $w=c x$ is in $\mathfrak{A}_{u}(1 / 2)$, and $w^{2}=1$.

For $x^{2}=\alpha+s$ where $s$ is nilpotent and $\alpha \neq 0$ is in $\mathfrak{F}$. Then $\beta=\left(\alpha^{-1}\right)^{1 / 2}$ is in $\mathfrak{F}$, and if $y=\beta x$, we have $y^{2}=\alpha^{-1}(\alpha+s)=1+z$ where $z=\alpha^{-1} s$ is nilpotent, $\mathfrak{F}[z] \subset \mathfrak{F}\left[x^{2}\right]$. Write

$$
c^{\prime}=(1+z)^{-1 / 2}=1-(1 / 2) z+(3 / 8) z^{2}+\cdots,
$$

where we use the fact that $z$ is nilpotent and the ordinary power series expansion of the function $(1+z)^{-1 / 2}$ to obtain a polynomial $c^{\prime}=c^{\prime}(z)$ with coefficients in $\mathfrak{F}$ such that $c^{\prime 2}(1+z)=1$. But $\mathfrak{F}[x]$ is associative and so $\left(c^{\prime} y\right)^{2}$ $=c^{\prime 2}(1+z)=1=(c x)^{2}$ where $c=\beta c^{\prime}, c x=c^{\prime} y$. Since $c$ is in $\mathcal{E}$ and $x$ is in $\mathfrak{A}_{u}(1 / 2)$,

(s) CPA, Lemma 10. 
the product $w$ is in $\mathfrak{A}_{u}(1 / 2)$.

The next step in our derivation is the substitution of the values

$$
x=k, \quad y=u, \quad z=w
$$

in (5), where

$$
k=k u, \quad w^{2}=1, \quad u w=(1 / 2) w .
$$

We also use that fact that $\mathscr{Q}$ is $u$-stable and so

$$
u(k w)=(1 / 2) k w .
$$

Then (5) becomes $4 k+4 w(k w)=2 w[u(k w)+k(u w)+w(u k)]+u[2 w(k w)$ $\left.+k w^{2}\right]+k\left[2(w u) w+u w^{2}\right]=4 w(k w)+2 u[w(k w)]+k+2 k$. This is equivalent to $k=2 u[w(k w)]$, a result which we state as follows.

Lemma 4. Let $c$ be in $\sqrt{5}$ so that $b=2 w[(c u) w]$ is in $\sqrt{ }$. Then $b u=c u$.

Consider the set $\mathfrak{Q}$ of all quantities $q$ in $\mathfrak{A}$ such that

$$
w(w q)=q .
$$

Evidently $\mathfrak{Q}$ is a subspace over $\mathfrak{F}$ of $\mathfrak{A}$. We determine $\mathfrak{Q}$ as follows.

Lemma 5. A quantity $q$ of $\mathfrak{A}$ is in $\mathfrak{Q}$ if and only if $q=(w x) w$ for $x$ in $\mathfrak{A}$. The intersection $\mathfrak{B}$ of $\mathfrak{Q}$ and $\mathfrak{E}$ is the set of all quantities $b=(w c) w$ for $c$ in $\mathfrak{E}$.

For proof we substitute $y=z=w$ in (5) to obtain $12\left[(w x) w^{2}\right]=12 w x$ $=3 w\left[2(w x) w+w^{2} x\right]+3 x w=6 w[(w x) w]+6 w x$. Thus our assumption about the characteristic of $\mathfrak{F}$ implies that

$$
w[(w x) w]=w x
$$

for every $x$ of $\mathfrak{A}$. Put $q=(w x) w$ and see that $w q=w x,(w q) w=(w x) w=q$. Thus every $x$ of $\mathfrak{A}$ determines a quantity $(w x) w$ in $\mathfrak{Q}$. Conversely if $q$ is in $\mathfrak{Q}$, we have $q=(w x) w$ for $x=q$. If $b$ is in $\mathscr{B}$, then $b=(w b) w$ where $b$ is in $\mathbb{E}$. Conversely if $c$ is in $\mathfrak{E}$, then $b=(w c) w$ is in $\mathfrak{Q}$. But $(w c) w$ is in $\mathfrak{A}_{u}(1 / 2) \mathfrak{A}_{u}(1 / 2) \subseteq \mathfrak{C}$ and so $b$ is in $\mathfrak{B}$, our proof is complete. Then

Lemma 6. Let $c$ be in $\mathfrak{c}$. Then $w c=0$ if and only if $c=b(u-v)$ for $b$ in $\mathfrak{B}$.

$$
w(b u)=w(b v)=(1 / 2) w b
$$

for every $b$ of $\mathfrak{B}$.

Let us write $a=2[w(b u)] w, c=2[w(b v)] w$ for $b$ in $\mathfrak{B}$ so that $a$ and $c$ are in $\mathfrak{B}$. By Lemma $4, a u=b u$, and by symmetry $b v=c v$. Then $a+c=2(w b) w$ $=2 b=2 b u+2 b v=a u+c u+a v+c v$. It follows that $b u+b v=a v+c u, a v=b v$. $a=a(u+v)=b=c$.

$$
2 w[w(b u)]=2 w[w(b v)]=b .
$$


Hence $w[w(b u-b v)]=0$. By (7) we obtain $w(b u-b v)=0$ and $w(b u)=w(b v)$, $w b=w(b u+b v)=2 w(b u)=2 w(b v)$, that is, (8) holds. Conversely if $c$ is in 5 and $w c=0$, then $b=2 w[w(c u)]$ is in $\mathfrak{B}, 2 w[w(c v)]=d$ is in $\mathfrak{B}, c u=b u, d v=c v$ by Lemma 4. But then $b+d=2 w(w c)=0, d=d u+d v=d u+c v=-b=-c u$ $-b v, c v=-b v, c=c(u+v)=b(u-v)$ as desired.

The quantities

$$
e=(1 / 2)(1+w), \quad f=(1 / 2)(1-w)=1-e
$$

are orthogonal idempotents and there is a corresponding decomposition

$$
\mathfrak{A}=\mathfrak{A}_{e}(1)+\mathfrak{A}_{e}(0)+\mathfrak{A}_{e}(1 / 2),
$$

where $\mathfrak{A}_{\theta}(0)=\mathfrak{A}_{f}(1)$. We propose to express this decomposition in terms of $w, \mathfrak{B}$, and the decomposition (1) of the algebra $\mathfrak{A}$ relative to $u$.

LEMмA 7. Let $\mathfrak{B}$ be the set of all elements $b$ of $\mathfrak{S}=\mathfrak{A}_{u}(1)+\mathfrak{A}_{u}(0)$ such that $(w b) w=b$. Then $\mathfrak{A}_{e}(1)=e \mathfrak{B}, \mathfrak{A}_{f}(1)=\mathfrak{A}_{e}(0)=f \mathfrak{B}, \mathfrak{A}_{e}(1 / 2)=(u-v) \mathfrak{B}+(\mathfrak{S})$ where (5) is the set of all elements $g$ of $\mathfrak{A}_{u}(1 / 2)$ such that $w g=0$. Also $\mathfrak{A}_{u}(1)=u \mathscr{B}, \mathfrak{A}_{u}(0)$ $=v \mathfrak{B}, \mathfrak{A}_{u}(1 / 2)=w \mathfrak{B}+\mathfrak{B}, \mathfrak{C}=\mathfrak{B}+(u-v) \mathfrak{B}$.

For every element of $\mathfrak{A}$ has the form $x=c+y$ where $c$ is in $\mathfrak{C}$ and $y$ is in $\mathfrak{A}_{u}(1 / 2)$. Then

$$
2 e x=(1+w) x=\mu x
$$

if and only if

$$
w x=(\mu-1) x
$$

Since $\mathfrak{A}$ is $u$-stable and (11) is equivalent to

$$
w c+w y=(\mu-1) c+(\mu-1) y,
$$

we see that

$$
w c=(\mu-1) y, \quad w y=(\mu-1) c .
$$

The value of $\mu$ for elements of $\mathfrak{A}_{\epsilon}(\lambda)$ is $\mu=2 \lambda$. Hence $x$ is in $\mathfrak{A}_{e}(1)$ if and only if $y=w c, w y=w(w c)=c$. Thus $c$ is in $\mathfrak{B}, x=(w+1) c=2 e c$, and we have proved that $\mathfrak{A}_{\odot}(1)=e \mathfrak{B}$. By symmetry $\mathfrak{A}_{f}(1)=\mathfrak{A}_{\odot}(0)=f \mathfrak{B}$. Also $x$ is in $\mathfrak{A}_{\odot}(1 / 2)$ if and only if $w c=w y=0, y$ is in $\mathbb{B}, c$ is in $\mathfrak{B}(u-v)$. This completes the expression of the decomposition of $\mathfrak{A}$ relative to $e$ in terms of $w, \mathfrak{B}$, and $\mathfrak{B}$.

Observe that if $c$ is in $\mathfrak{E}$, then $(w c) w=b$ is in $\mathfrak{B},[w(c-b)] w=0, c-b$ $=a(u-v)$ for $a$ in $\mathfrak{B}, c=b+a(u-v), \mathfrak{S}=\mathfrak{B}+\mathfrak{B}(u-v)$. Clearly $\mathfrak{A}_{u}(1)=u \mathfrak{C}$ $=u \mathfrak{B}, \mathfrak{A}_{u}(0)=v \mathfrak{B}$. Since every $x$ of $\mathfrak{A}$ has the form $e a+f b+(u-v) c+g$ with $a, b, c$ in $\mathfrak{B}$ and $g$ in $(\mathfrak{S}$, we see that $x=(1 / 2)(a+b)+(1 / 2) w(a-b)+(u-v) c+g$ and the component of $x$ in $\mathfrak{P}_{u}(1 / 2)$ is $(1 / 2) w(a-b)+g$. But then $\mathfrak{A}_{u}(1 / 2) \subseteq w \mathfrak{B}$ $+B \subseteq \mathfrak{A}_{u}(1 / 2)$ and we have the desired result.

We shall actually show that $\mathfrak{B}$ is a subalgebra of $\mathfrak{E}$, and it will follow that 
$\mathfrak{A}_{u}(1)$ and $\mathfrak{A}_{u}(0)$ are isomorphic to $\mathfrak{B}$. The next step in our derivation of this vital result is the use of some known $\left(^{(6)}\right.$ results which we write as

$$
\begin{gathered}
w[(a b) u]=w[(a u)(b u)]=[w(a u)](b u)+[w(b u)](a u), \\
{[w(a u)](b v)=[w(b v)](a u),}
\end{gathered}
$$

and the result of replacing $u$ by $v$ in (13). These equations hold for all quantities $a$ and $b$ of $\mathbb{E}$ and we take $a$ and $b$ in $\mathscr{B}$ so that $w(a u)=w(a v), w(b u)=w(b v)$ by the proof of Lemma 6. Then $(w a) b=[w(a u+a v)](b u+b v)=[w(a u)](b u)$ $+[w(a u)](b v)+[w(a v)](b v)+[w(a v)](b u)=[w(a u)](b u)+[w(b v)](a u)$ $+[w(a v)](b v)+[w(b u)](a v)=w[(a b) u+(a b) v]=w(a b)$. Also $[w(b u+b v)](a u$ $-a v)=2[w(b v)](a u)-2[w(b u)](a v)=2[w(a u)](b v)-2[w(a v)](b u)=(w a)(b v$ $-b u)$. We have proved the following result.

Lemma 8. Let $a$ and $b$ be in $\mathfrak{B}$. Then $(w a) b=w(a b),(w b)[a(u-v)]$ $=-(w a)[b(u-v)]$.

The substitution $x=w, y=a, z=b$ in (5) yields $4[2(w a)(w b)+a b]$ $=2 w[(w a) b+(w b) a+w(a b)]+a\left[2 w(w b)+w^{2} b\right]+b\left[2 w(w a)+w^{2} a\right]=6 w[w(a b)]$ $+6 a b, 8(w a)(w b)=6 w[w(a b)]+2 a b$,

$$
4(w a)(w b)=3 w[w(a b)]+a b .
$$

Since $\mathfrak{H}_{e}(1)$ is an algebra, we have $(a+w a)(b+w b)=2(d+w d)$ for every $a$ and $b$ of $\mathfrak{B}$ where $d$ is in $\mathfrak{B}$. Then $a b+(w a)(w b)+(w a) b+(w b) a=a b+(w a)(w b)$ $+2 w(a b)=2 d+2 w d$,

$$
a b+(w a)(w b)=2 d, \quad w(a b)=w d .
$$

Then $4(w a)(w b)=8 d-4 a b=3 w[w(a b)]+a b$. Since both $d$ and $w[w(a b)]$ are in $\mathfrak{B}$, so is $5 a b=8 d-3 w[w(a b)]$. Thus $a b$ is in $\mathscr{B}$ and $\mathfrak{B}$ is a subalgebra of $\mathfrak{E}$. Also $w[w(a b)]=a b$ so that (15) implies that

$$
(w a)(w b)=a b
$$

for every $a$ and $b$ of $\mathfrak{B}$. It is now true that $(e a)(e b)=e d=e(a b)$ and $\mathfrak{A}_{e}(1)$ is isomorphic to $\mathfrak{B}$. We are now ready to show that $\mathfrak{A}_{u}(1)$ is isomorphic to $\mathfrak{B}$.

THEOREM 2. Let $\mathfrak{A}$ be a simple commutative power-associative algebra of degree two over an algebraically closed field $\mathfrak{F}$ whose characteristic is prime to 30 so that $\mathfrak{A}$ has a unity quantity 1 and $1=u+v$ where $u$ is an idempotent, $v \neq 0$. Then if $\mathfrak{A}$ is $u$-stable, its subalgebras $\mathfrak{A}_{u}(1)$ and $\mathfrak{A}_{u}(0)$ are isomorphic.

For $\mathfrak{A}_{u}(1)=u \mathfrak{E}=u[\mathfrak{B}+(u-v) \mathfrak{B}]=u \mathfrak{B}$. The mapping $b \rightarrow b u$ is a one-to-one mapping of $\mathfrak{B}$ onto $\mathfrak{A}_{u}(1)$ since $2 w[w(b u)]=w(w b)=b$. Since $(a b) u=(a u)(b u)$ for every $a$ and $b$ of $\mathfrak{B}$ (and indeed for every $a$ and $b$ of ()), the mapping is an

(6) Formulas (5) and (7) of CPA are equivalent to (13) and (14) in the $u$-stable case. The proofs of CPA can be simplified considerably in the stable case. 
isomorphism. By symmetry $\mathfrak{B}$ is isomorphic to $\mathfrak{A}_{v}(1)=\mathfrak{A}_{u}(0)$ and so $\mathfrak{A}_{u}(1)$ and $\mathfrak{A}_{u}(0)$ are isomorphic.

The algebra $\mathfrak{B}=\mathfrak{F}+\mathfrak{N}$ where $\mathfrak{N}$ is the radical of $\mathfrak{B}$ and is isomorphic to both $\mathfrak{N}_{0}$ and $\mathfrak{R}_{1}$. Evidently

$$
\mathfrak{N}^{\prime}=\mathfrak{N}_{1} \oplus \mathfrak{N}_{0}=\mathfrak{N}+(u-v) \mathfrak{R} .
$$

Since $x(u-v)=0$ for every $x$ of $\mathfrak{A}_{u}(1 / 2)$, we see that if

$$
b=\beta+b^{*}
$$

is any quantity of $\mathfrak{B}$, then

$$
x[b(u-v)]=x\left[b^{*}(u-v)\right]
$$

for every $x$ of $\mathfrak{A}_{u}(1 / 2)$. By Lemma 8 we have:

Lemma 9. Let $a=\alpha+a^{*}$ and $b=\beta+b^{*}$ be in $\mathfrak{B}$ so that $a^{*}$ and $b^{*}$ are in $\mathfrak{N}$ and $\alpha$ and $\beta$ are in $\mathfrak{F}$. Then $(w b)[a(u-v)]=\left(w b^{*}\right)\left[a^{*}(u-v)\right]$.

The result above actually follows directly from Lemma 9 since $(w b)[a(u-v)]=\left(w b^{*}\right)[a(u-v)]+\beta w[a(u-v)]$ and we use (17) and Lemma 6.

We shall close our study of $u$-stable algebras by deriving a sequence of lemmas which we shall use in a determination of all stable algebras.

Lemma 10. Let $b$ be in $\mathfrak{B}$ and $g$ be in (5). Then

$$
g[(u-v) b]=w d
$$

where $d$ is in $\mathfrak{B}$. Also $(w b)[a(u-v)]$ is in $\mathbb{H}$ for every a and $b$ of $\mathfrak{B}$.

For $\left[\mathfrak{A}_{e}(1 / 2)\right]^{2} \subseteq \mathfrak{A}_{e}(1)+\mathfrak{A}_{e}(0)$ and so $g[(u-v) b]=(1 / 2)(d+w d)+(1 / 2)$ $\cdot(c-w c)$ for $d$ and $c$ in $\mathfrak{B}$. Since $g[(u-v) b]$ is in $\mathfrak{A}_{u}(1 / 2)$ we have $c+d=0$, and (18) is proved. The relation $\mathfrak{A}_{e}(1) \mathfrak{A}_{e}(1 / 2) \subseteq \mathfrak{A}_{e}(1 / 2)+\mathfrak{A}_{e}(0)$ implies that $(b+w b)[a(u-v)]=c(u-v)+g+d-w d$ where $c$ and $d$ are in $\mathfrak{B}$ and $g$ is in $(S)$. Then $b[a(u-v)]=(b a)(u-v)=c(u-v)+d, d=(b a-c)(u-v)$ is in $\mathfrak{B}$ and in $(u-v) \mathfrak{B}, d=b a-c=0$, $(w b)[a(u-v)]=g$ is in $(S)$.

LEMMA 11. The product gh of any two elements of $(B)$ is in $\mathfrak{B}$.

For we put $x=y=g$ and $z=w$ in (5) and obtain $4 g^{2}=2 w\left[2 g(w g)+w g^{2}\right]$ $+2 g\left[2 w(w g)+w^{2} g\right]=2 w\left(w g^{2}\right)+2 g^{2}$ since $w g=0, w^{2}=1$. Thus $g^{2}=w\left(g^{2} w\right)$. However $g^{2}$ is in $\mathbb{S}$ for every $g$ of $\mathfrak{A}_{u}(1 / 2)$ and so $g^{2}$ is in $\mathfrak{B}$ for every $g$ of $\mathfrak{S}$. Clearly $2 g h=(g+h)^{2}-g^{2}-h^{2}$ is in $\mathfrak{B}$ for every $g$ and $h$ of the vector space $\mathfrak{S}$, $g h$ is in $\mathfrak{B}$.

Lemмa 12. Let $g$ be in $\mathfrak{B S}$ and $b$ be in $\mathfrak{B}$ so that (18) holds for $d$ in $\mathfrak{B}$. Then

$$
g b=h-w c
$$

for $h$ in $(S)$ and $c$ in $\mathfrak{B}$, and 


$$
w(g b)=-c, \quad(w b) g+w(g b)=-d(u-v) .
$$

Equation (19) is simply an application of the fact that $\mathfrak{A}_{u}(1 / 2)=w \mathscr{B}+(\mathfrak{S}$. Now $(e \mathfrak{B})\left(s \subseteq \mathfrak{A}_{\theta}(1 / 2)+\mathfrak{A}_{e}(0)\right.$ and so $(b+w b) g=a(u-v)+h+c-w c$ for $a$ and $c$ in $\mathfrak{B}$ and $h$ in $(5)$. This yields $g b=h-w c$ and $(w b) g=a(u-v)+c$ and these results are trivial. They imply that $w(g b)=-w(c w)=-c$ so that $(w b) g$ $+w(g b)=a(u-v)$. To prove that $a=-d$ we substitute $x=g, y=b, z=u-v$ in (5) and obtain $4[(b z)(w g)+(b w)(z g)+(b g)(w z)]=0=g[b(z w)+(b z) w+(b w) z]$ $+b[(z g) w+z(g w)+(w z) g]+(u-v)[g(b w)+(g b) w+b(g w)]+w[(b g) z+g(b z)$ $+(g z) b]=w[g(b z)]+(u-v)[g(b w)+(g b) w]$. Using $[(w b) g+w(g b)](u-v)$ $=[a(u-v)](u-v)=a$ and $w[g(b z)]=w(w d)$ we have

$$
[(w b) g+w(g b)](u-v)=-w[g(b u-b v)]=-d .
$$

This completes our set of properties and we pass on to the stable case.

3. Stable algebras. The concept of stability is vacuous for simple algebras of degree one. If $\mathfrak{A}$ is a central simple algebra of degree $t>1$ over its center $\mathfrak{F}$, then $\mathfrak{A}$ is stable if and only if $\mathfrak{A}_{\mathfrak{R}}$ is a central simple stable algebra for every scalar extension $\mathfrak{R}$ of $\mathfrak{F}$. Moreover $\mathfrak{A}$ is a Jordan algebra if and only if every $\mathfrak{A}_{\mathfrak{R}}$ is a Jordan algebra. Since $\mathfrak{A}$ is a Jordan algebra if $t>2$, the proof of the theorem of our introduction can be reduced to the proof in the case where $t=2$ and $\mathfrak{F}$ is algebraically closed.

We now let $\mathfrak{A}$ be stable so that all of the properties of $\$ 2$ will hold and $\mathfrak{A}$ will also be $e$-stable. Then (19) holds with $c=0$. This yields:

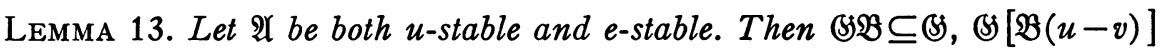
$\subseteq w \mathfrak{B}$. Moreover, if $b$ is in $\mathfrak{B}$ and $g$ is in $(S)$ so that $g(b u-b v)=w d$ for $d$ in $\mathfrak{B}$, then $(w b) g=-d(u-v)$.

We shall now strengthen the result of Lemma 13.

LEMma 14. The product $\leftrightarrow[\mathfrak{B}(u-v)] \subseteq w \Re$ and $(w \mathfrak{B})(S \subseteq \Re(u-v)$.

For Lemma 13 implies that $-(w b) g=d(u-v)=\delta(u-v)+d^{*}(u-v)$ for $\delta$ in $\mathfrak{F}$ and $d^{*}$. in $\mathfrak{R}$. But Lemma 1 implies that $(w b) g=\alpha(u+v)+k$ for $k$ in $\mathfrak{N}^{\prime}$. Hence $\alpha=\delta=-\delta=0$ and $d=d^{*}$ is in $\mathfrak{R}$. Our result is then an immediate consequence of Lemma 13.

The following property is proved quite trivially.

LEMmA 15. The product $\left[\mathfrak{N}^{\prime} \mathfrak{A}_{u}(1 / 2)\right] \mathfrak{E} \subseteq \mathfrak{N}^{\prime} \mathfrak{A}_{u}(1 / 2)$.

Let $k$ be in $\mathfrak{N}^{\prime}$ and $x$ be in $\mathfrak{A}_{u}(1 / 2)$. Then if $b=\beta+b^{*}$ is any quantity of $\mathfrak{B}$, we have $\beta$ in $\mathfrak{F}, b^{*}$ in $\mathfrak{N},(x k) b=\beta x k+(x k) b^{*}$ in $\mathfrak{N}^{\prime} \mathfrak{A}_{u}(1 / 2)$ since $x k$ is in $\mathfrak{N}^{\prime} \mathfrak{P}_{u}(1 / 2), x k$ is in $\mathfrak{A}_{u}(1 / 2),(x k) b^{*}$ is in $\mathfrak{N A}_{u}(1 / 2)$. It remains to show that $(x k)[b(u-v)]=(x k)\left[b^{*}(u-v)\right]+\beta(x k)(u-v)=(x k)\left[b^{*}(u-v)\right]$ is in $\mathfrak{N}^{\prime} \mathfrak{A}_{u}(1 / 2)$ and this quantity is clearly in $[\mathfrak{R}(u-v)] \mathfrak{A}_{u}(1 / 2)$, a subspace of $\mathfrak{N}^{\prime} \mathfrak{A}_{u}(1 / 2)$ 
$=\mathfrak{N \mathfrak { A } _ { u }}(1 / 2)+[\mathfrak{N}(u-v)] \mathfrak{A}_{u}(1 / 2)$.

Let us substitute $x=g, y=a, z=b(u-v)$ in (5) and use the properties $w z=w g=0$ to obtain $4(w a)(g z)=w[a(z g)+(a z) g+(a g) z]+a[w(z g)]+z[(w a) g$ $+w(a g)]+g[(w a) z]$, where $g[w(a z)]=0$ by Lemma 6 . The quantity $z g$ $=g(b u-b v)=w d^{*}$ where $d^{*}$ is in $\mathfrak{N}$ by Lemma $14, w(z g)$ is in $\Re, a[w(z g)]$ is in $\mathfrak{R}$ for every $a$ of $\mathfrak{B}$. Also $a(z g)=w\left(d^{*} a\right), w[a(z g)]=d^{*} a$ is in $\mathfrak{N}$, $a z$ $=(a b)(u-v),(a z) g$ is in $w \mathfrak{N}$ by Lemma $14, w[(a z) g]$ is in $\mathfrak{N}$. The quantity $a g$ is in $\$,(a g) z$ is in $w \Re$ by Lemma $14, w[(a g) z]$ is in $\Re$. The quantity $w(a g)=0$ by Lemma 13, (wa)g is in $\mathfrak{N}(u-v), z[(w a) g]$ is in $\mathfrak{N}$. Thus $g[(w a) z]$ is in $\mathfrak{N}$ and we state this result as follows.

Lemma 16. The product $\mathfrak{B}\{(w \mathfrak{B})[\mathfrak{B}(u-v)]\} \subseteq \mathfrak{N}$.

We are now ready to prove the critical result of the proof of the theorem of our introduction.

Lemma 17. The product $\left[\mathfrak{R}^{\prime} \mathfrak{A}_{u}(1 / 2)\right] \mathfrak{A}_{u}(1 / 2) \subseteq \mathfrak{N}^{\prime}$.

We first consider the element $t=w a$ where $a$ is in $\mathfrak{B}$. If $b$ is in $\Re$, we have $t b=(w a) b=w(a b)=w d^{*}$ where $d^{*}$ is in $\mathfrak{N},(t b)(w c)=c d^{*}$ is in $\mathfrak{N}$ for every $c$ of $\mathfrak{B},(t \mathfrak{N})(w \mathfrak{B}) \subseteq \mathfrak{N}$. Also $(t \mathfrak{N})\left(\mathfrak{S} \subseteq \mathfrak{N}^{\prime}\right.$ by Lemma 14 and so $(t \mathfrak{N}) \mathfrak{A}_{u}(1 / 2) \subseteq \mathfrak{N}^{\prime}$.

We next write $s=t[b(u-v)]$. By Lemma 16 we know that $s(\mathfrak{s} \subseteq \mathfrak{R}$. But $s$ is in $\&$ by Lemma 10 and so $s(w b) \subseteq \mathfrak{R}^{\prime}$ by Lemma 14 . Hence $\left(t \mathfrak{R}^{\prime}\right) \mathfrak{A}_{u}(1 / 2)$ $\subseteq \mathfrak{N}^{\prime}$ for every $t$ in w\&.

Suppose now that $t$ is any nonsingular element of $\mathfrak{A}_{u}(1 / 2)$. We apply Lemma 3 to show that there exists an element $c$ in $\mathfrak{F}\left[t^{2}\right]$ such that $w=c t$ has the property that $w^{2}=1$. Then $t=c^{-1} w=d w$ where $d$ is in $\mathfrak{F}\left[t^{2}\right] \subseteq \mathfrak{E}$. Then $d=a+b(u-v)$ where $a$ and $b$ are in the algebra $\mathfrak{B}$ defined for this $w, d w=a w$ $+w[b(u-v)]=a w=t$ by Lemma 6 . Thus $\left(t \mathfrak{N}^{\prime}\right) \mathfrak{A}_{u}(1 / 2) \subseteq \mathfrak{N}^{\prime}$ for every nonsingular element $t$ of $\mathfrak{A}_{u}(1 / 2)$. But if $t_{1}$ is a singular element of $\mathfrak{A}_{u}(1 / 2)$, there exists an element $\alpha$ in $\mathfrak{F}$ such that $t=\alpha w+t_{1}$ is nonsingular, $\left(t_{1} \mathfrak{N}^{\prime}\right) \mathfrak{A}_{u}(1 / 2)$ $\subseteq\left(t \mathfrak{N}^{\prime}\right) \mathfrak{A}_{u}(1 / 2)+\left(w \mathfrak{N}^{\prime}\right) \mathfrak{A}_{u}(1 / 2) \subseteq \mathfrak{N}^{\prime}$ since $w$ and $t$ are nonsingular. It follows that $\left[\mathfrak{N}^{\prime} \mathfrak{A}_{u}(1 / 2)\right] \mathfrak{H}_{u}(1 / 2) \subseteq \mathfrak{N}^{\prime}$ as desired. This completes our proof of Lemma 17.

We may now write $\mathfrak{M}=\mathfrak{R}^{\prime}+\mathfrak{A}_{u}(1 / 2) \mathfrak{R}^{\prime}$. By Lemma 17 we know that $\left[\mathfrak{N}^{\prime} \mathfrak{A}_{u}(1 / 2)\right] \mathfrak{A}_{u}(1 / 2) \subseteq \mathfrak{R}^{\prime} \subseteq \mathfrak{M}$. Also $\left[\mathfrak{R}^{\prime} \mathfrak{A}_{u}(1 / 2)\right] \subseteq \subseteq \mathfrak{N}^{\prime} \mathfrak{A}_{u}(1 / 2)$ by Lemma 15 and so $\left[\mathfrak{N}^{\prime} \mathfrak{A}_{u}(1 / 2)\right] \mathfrak{U} \subseteq \mathfrak{M}$. But $\mathfrak{N}^{\prime} \mathfrak{A}=\mathfrak{N}^{\prime} \mathfrak{C}+\mathfrak{N}^{\prime} \mathfrak{A}_{u}(1 / 2) \subseteq \mathfrak{M}$ and we have shown that $\mathfrak{M}$ is an ideal of $\mathfrak{A}$. Clearly 1 is not in $\mathfrak{M}$ and the hypothesis that $\mathfrak{A}$ is simple implies that $\mathfrak{M}=\mathfrak{N}^{\prime}=0$. Then $\mathfrak{A}=u \mathfrak{F}+v \mathfrak{F}+\mathfrak{A}_{u}(1 / 2)=\mathfrak{F}+u_{0} \mathfrak{F}$ $+\mathfrak{A}_{u}(1 / 2)$ where $u_{0}=u-v, u_{0} x_{0}=0$ for every $x_{0}$ of $\mathfrak{A}_{u}(1 / 2)$. By Lemma 1 we know that $x_{0}^{2}=\alpha_{0}$ in $\mathfrak{F}$ for every $x_{0}$ of $\mathfrak{A}_{u}(1 / 2)$. If $x=\lambda+\mu u_{0}+\nu x_{0}$ is any quantity of $\mathfrak{A}$ we see that $x^{2}-2 \lambda x+\lambda^{2}=\mu^{2}+\nu^{2} \alpha_{0}$ and so $x^{2}=\zeta x+\eta$ where $\zeta$ and $\eta$ are in $\mathfrak{F}$. But then $x\left(y x^{2}\right)-(x y) x^{2}=\zeta[x(y x)-(x y) x]+\eta(x y-y x)=0$ and $\mathfrak{A}$ is a Jordan algebra. This completes our proof of Theorem 1 .

4. The derivations of the algebras of Kokoris. A derivation of an algebra 
$\mathfrak{S}$ over a field $\mathfrak{F}$ is a linear transformation $s \rightarrow s D$ over $\mathfrak{F}$ of $\subseteq$ such that

$$
(r s) D=(r D) s+r(s D)
$$

for every $r$ and $s$ of $\mathfrak{S}$. The algebra $\mathfrak{D}$ of all derivations of $\mathfrak{S}$ is a Lie algebra with respect to the product operation

$$
D_{1} \circ D_{2}=D_{1} D_{2}-D_{2} D_{1}
$$

where $D_{1} D_{2}$ and $D_{2} D_{1}$ are the ordinary linear transformation products.

Let $\mathfrak{B}=\mathfrak{F}[1, x]$ be the algebra of all polynomials $b=b(x)$ with coefficients in $\mathfrak{F}$ in $1=x^{0}$ and $x$, where $x^{p}=0, \mathfrak{B}$ has dimension $p$ over $\mathfrak{F}$, and $\mathfrak{F}$ has characteristic $p$. The derivation algebra of $\mathfrak{B}$ is the well known simple Lie algebra of Witt. We shall designate this algebra by $\mathfrak{B}$. It consists of all linear transformations $D(c)$ where $c$ is any quantity of $\mathscr{B}$ and $D(c)$ is the transformation

$$
b=b(x) \rightarrow b D(c)=b^{\prime}(x) c(x) .
$$

Here $b^{\prime}(x)$ is the ordinary derivative of $b(x)$, and

$$
D(a) \circ D(c)=D\left(a^{\prime} c-a c^{\prime}\right),
$$

for every $a$ and $c$ of $\mathfrak{B}$.

The algebra $\mathfrak{S}$ of Kokoris may be defined as follows. Let $\mathfrak{B}$ be imbedded in the vector space

$$
\mathfrak{B}+\mathfrak{B u}+y_{0} \mathfrak{B}+y_{1} \mathfrak{B}
$$

of dimension $4 p$ over $\mathfrak{F}$, where

$$
\mathfrak{S}=\mathfrak{B}+\mathfrak{B} u=\mathfrak{B} u \oplus \mathfrak{B} v
$$

is the associative algebra which is the direct sum of $B u$ and $B v$ for an idempotent $u \neq 1$ and $v=1-u$. Then $a+b u+y_{0} c+y_{1} d=0$ for $a, b, c, d$ in $\mathfrak{B}$ if and only if $a=b=c=d=0$. We assume that

$$
\left(y_{i} a\right) b=y_{i}(a b)=2\left[y_{i}(a u)\right] b=2\left(y_{i} a\right)(u b) \quad(i=0,1),
$$

for every $a$ and $b$ of $\mathfrak{B}$. We also assume thait

$$
\left(y_{i} a\right)\left(y_{i} b\right)=0, \quad\left(y_{0} a\right)\left(y_{1} b\right)=a^{\prime} b-b^{\prime} a \quad(i=0,1),
$$

for every $a$ and $b$ of $\mathfrak{B}$ where $a^{\prime}$ and $b^{\prime}$ are the ordinary derivatives. The commutative law will then complete the definition of $\mathfrak{S}$. This algebra clearly contains the subalgebra

$$
\mathfrak{T}=\mathfrak{B}+y_{0} \mathfrak{B}+y_{1} \mathfrak{B},
$$

and both $\mathfrak{S}$ and $\mathfrak{T}$ are power-associative and central simple over $\mathfrak{F}$. We observe that $\mathfrak{S}=\mathfrak{S}_{u}(1) \oplus \mathfrak{S}_{u}(0)$ is the vector space direct sum

$$
\mathfrak{S}=\mathfrak{B}+(u-v) \mathfrak{B},
$$


and that

$$
y_{i}[(u-v) b]=0 \quad(i=0,1),
$$

for every $b$ of $\mathfrak{B}$.

We shall determine all derivations $D$ of $\mathfrak{S}$ and of $\mathfrak{T}$. Every such derivation is a linear transformation

$$
s \rightarrow s D=f(s)+(u-v) g(s)+y_{0} f_{0}(s)+y_{1} f_{1}(s),
$$

where $f(s), g(s), f_{0}(s), f_{1}(s)$ are linear functions of $s$ with values in $\mathscr{B}$ and $g(s)=0$ if $D$ is a derivation of $\mathfrak{T}$. Since

$$
\left(s^{2}\right) D=2 s(s D),
$$

for every $s$ of $\mathfrak{S}$, we see that (29) implies that

$$
\left(y_{i} b\right)\left[\left(y_{i} b\right) D\right]=0 \text {. }
$$

Then $y_{i}\left[b f\left(y_{i} b\right)\right]+(-1)^{i}\left\{b^{\prime} f_{1-i}\left(y_{i} b\right)-b\left[f_{1-i}\left(y_{i} b\right)\right]^{\prime}\right\}=0$ and so

$$
b f\left(y_{i} b\right)=0, \quad b^{\prime} f_{1-i}\left(y_{i} b\right)=b\left[f_{1-i}\left(y_{i} b\right)\right]^{\prime} .
$$

When $b$ is a nonsingular element of $\mathfrak{B}$, we see that (35) implies that $f\left(y_{i} b\right)=0$. If $b$ is singular, then $c=1+b$ is nonsingular, $f\left(y_{i} c\right)=f\left(y_{i}\right)=0, f\left(y_{i} b\right)=f\left(y_{i} c\right)$ $-f\left(y_{i}\right)=0$. We have proved the following result.

\section{Lemma 18. Let $D$ be a derivation of $\mathfrak{\$}$. Then}

$$
\left(y_{i} b\right) D=y_{0} f_{0}\left(y_{i} b\right)+y_{1} f_{1}\left(y_{i} b\right)+(u-v) g\left(y_{i} b\right)
$$

for every $b$ of $\mathfrak{B}$ where $f_{0}\left(y_{i} b\right), f_{1}\left(y_{i} b\right), g\left(y_{i} b\right)$ are linear functions of $y_{i} b$ with values in $\mathfrak{B}$ such that $b^{\prime} f_{1-i}\left(y_{i} b\right)=b\left[f_{1-i}\left(y_{i} b\right)\right]^{\prime}$. If $D$ is a derivation of $\mathfrak{T}$, then (36) holds with $g\left(y_{i} b\right)=0,\left(y_{i} b\right) D$ is in $y_{0} \mathfrak{B}+y_{1} \mathfrak{B}=\Im_{u}(1 / 2)$.

Lemma 18 implies that $\left[f_{0}\left(y_{1}\right)\right]^{\prime}=\left[f_{1}\left(y_{0}\right)\right]^{\prime}=0$. Thus we may write

$$
y_{0} D=g_{0}(u-v)+y_{0} f_{0}+\alpha y_{1}, \quad y_{1} D=g_{1}(u-v)+\beta y_{0}+y_{1} f_{1},
$$

where $g_{0}, g_{1}, f_{0}, f_{1}$ are in $\mathfrak{B}$ and $\alpha$ and $\beta$ are in $\mathfrak{F}$. Then $\left(y_{0} x\right) D=y_{0}(x D)+\left(y_{0} D\right) x$ $=y_{0}\left[f(x)+y_{0} f_{0}(x)+y_{1} f_{1}(x)\right]+g_{0} x(u-v)+y_{0} f_{0} x+\alpha y_{1} x$. The component in $\mathfrak{B}$ is $f\left(y_{0} x\right)=-\left[f_{1}(x)\right]^{\prime}=0$ by Lemma 18 . The computation of $\left(y_{1} x\right) D$ yields $\left[f_{0}(x)\right]^{\prime}=0$. Hence $x D=f(x)+(u-v) g(x)+\delta y_{0}+\epsilon y_{1}$ where $\delta$ and $\epsilon$ are in $\mathfrak{F}$. But $x^{2} D=2 x(x D)=2 x f(x)+2(u-v) x g(x)+2 \delta y_{0} x+2 \epsilon y_{1} x$ and so $\left(y_{0} x^{2}\right) D$ $=2 y_{0}[x(x D)]+\left(y_{0} D\right) x^{2}=2 y_{0}[x f(x)]+2 \epsilon y_{0}\left(y_{1} x\right)+\left[g_{0}(u-v)+y_{0} f_{0}+\alpha y_{1}\right] x^{2}$. Then $f\left(y_{0} x^{2}\right)=-2 \epsilon=0$. By symmetry the computation of $\left(y_{1} x^{2}\right) D$ yields $\delta=0$. We state the result as follows.

Lemma 19. If $D$ is a derivation of $\mathfrak{S}$ the quantity $x D$ is in $\mathfrak{S}=\mathfrak{B}+(u-v) \mathfrak{B}$. If $D$ is a derivation of $\mathfrak{T}$, then $x D$ is in $\mathfrak{B}$.

Since $u^{2}=u$, we have $2 u(u D)=u D$ and so $u D$ is in $y_{0} \mathfrak{B}+y_{1} \mathfrak{B}$ and we may 
write

$$
u D=y_{0} c_{0}+y_{1} c_{1},
$$

where $c_{0}$ and $c_{1}$ are in $\mathscr{B}$. The relation $u y_{0}=(1 / 2) y_{0}$ yields $(1 / 2) y_{0} D=u\left(y_{0} D\right)$ $+(u D) y_{0}=g_{0} u+(1 / 2)\left(y_{0} f_{0}+\alpha y_{1}\right)-c_{1}^{\prime}=(1 / 2)\left[g_{0}(u-v)+y_{0} f_{0}+\alpha y_{1}\right]$. Thus $c_{1}^{\prime}$ $=(1 / 2) g_{0}(u+v)$ and so

$$
2 c_{1}^{\prime}=g_{0} .
$$

Similarly $(1 / 2) y_{1} D=u\left(y_{1} D\right)+(u D) y_{1}=g_{1} u+(1 / 2)\left(\beta y_{0}+y_{1} f_{1}\right)+c_{0}^{\prime}=(1 / 2)\left[g_{1}(u\right.$ $\left.-v)+\beta y_{0}+y_{1} f\right]$ and we obtain

$$
2 c_{0}^{\prime}=-g_{1} .
$$

Let us introduce the notation

$$
(u x) D=d u+d_{2}+z
$$

where $d$ and $d_{2}$ are in $B$ and $u z=(1 / 2) z$. Since $u(u x)=u x$, we conclude that $d u+d_{2}+z=u[(u x) D]+(u D)(u x)=d u+d_{2} u+(1 / 2) z+(1 / 2) x(u D)$. Thus $z$ $=x(u D)$ and $d_{2} v=0, d_{2}=0$,

$$
(u x) D=d u+x(u D) .
$$

Also $(v x) D=e v+e_{2}+t$ where $e$ and $e_{2}$ are in $B$ and $u t=(1 / 2) t$. Since $u x+v x=x$ and $x D$ is in $\mathfrak{E}$, we conclude that $t=-x(u D)$. However, by symmetry, the fact that $v(v x)=v x$ implies that $e_{2} u=e_{2}=0$ and so

$$
(v x) D=e v-x(u D) .
$$

We now use the fact that $y_{i}(x u)=y_{i}(x v)$. Then $y_{0}[(x u) D]+\left(y_{0} D\right)(x u)$ $=y_{0}[(x v) D]+\left(y_{0} D\right)(x v), \quad\left(y_{0} D\right)(x u-x v)=\left[g_{0}(u-v)\right] x(u-v)=g_{0} x=y_{0}[(x v) D$ $-(x u) D]=y_{0}[e v-d u-2 x(u D)]$. But then $y_{0}(e v-d u)=(1 / 2) y_{0}(e-d)=0$ and so $e=d, x D=d(u+v)=d$ is in $\mathscr{B}$. Also $g_{0} x=-2 y_{0}\left[\left(y_{0} c_{0}+y_{1} c_{1}\right) x\right]=2\left(c_{1} x\right)^{\prime}$ $=2\left(c_{1}+x c_{1}^{\prime}\right)=2 c_{1}+g_{0} x$ by (38). Thus $2 c_{1}=0, g_{0}=2 c_{1}^{\prime}=0$. We similarly see that $\left(y_{1} D\right)(x u-x v)=g_{1} x=y_{1}[(x v) D-(x u) D]=-2 y_{1}[x(u D)]=-2 y_{1}\left[y_{0}\left(c_{0} x\right)\right]$ $=-2\left(c_{0} x\right)^{\prime}=-2 c_{0}+g_{1} x, c_{0}=0, g_{1}=0$. We have proved the following result.

Lemma 20. Let $D$ be any derivation of $\mathfrak{S}$. Then $u D=0, x D$ is in $\mathfrak{B}, y_{0} D$ $=y_{0} f_{0}+\alpha y_{1}, y_{1} D=\beta y_{0}+y_{1} f_{1}$ where $\alpha$ and $\beta$ are in $\mathfrak{F}, f_{0}$ and $f_{1}$ are in $\mathfrak{B}$.

We are now ready to establish the following theorem.

THEOREM 3. Every derivation $D$ of $S=\mathfrak{B}+\mathfrak{B} u+y_{0} \mathfrak{B}+y_{1} \mathfrak{B}$ induces a derivation $t \rightarrow t D$ of $\mathfrak{T}$. Conversely every derivation $D$ of $\mathfrak{T}$ may be extended to a derivation $D$ of $\subseteq$ with $(b u) D=b(u D)$ for every $b$ of $\mathfrak{B}$. Thus $\subseteq$ and $\mathfrak{T}$ have isomorphic derivation algebras.

For Lemmas 18 and 20 imply that $y_{i} D$ is in $\Im_{u}(1 / 2)=y_{0} \mathfrak{B}+y_{1} \mathfrak{F}$ for 
every derivation $D$ of either $\mathfrak{S}$ or $\mathfrak{T}$. Also Lemmas 19 and 20 imply that $x D$ is in $\mathfrak{B}$ for every $D$ of either $\mathfrak{S}$ or $\mathfrak{T}$. But if $x^{k} D=k x^{k-1}(x D)$, then $x^{k+1} D$ $=\left(x^{k} D\right) x+x^{k}(x D)=k\left[x^{k-1}(x D)\right] x+x^{k}(x D)=(k+1) x^{k}(x D)$ since $x D$ is in the associative algebra $\mathfrak{B}$. Thus

$$
b D=b^{\prime}(x D)
$$

for every $b$ of $\mathfrak{B}$ and every derivation $D$ of either $\mathfrak{S}$ or $\mathfrak{T}$.

If $s \rightarrow s D$ is a derivation of $\mathfrak{S}$, it should now be clear that $t D$ is in $\mathfrak{T}$ for every $t$ of $\mathfrak{T}$ and so $t \rightarrow t D$ is a derivation of $\mathfrak{T}$. Conversely let $D$ be a derivation of $\mathfrak{T}$. We extend $D$ to a linear transformation of $\subseteq$ by writing $(b u) D$ $=(b D) u$ for every $b$ of $\mathfrak{B}$ where $D$ thus maps $u \mathscr{B}$ into itself. It remains to show that (22) holds for every $r$ and $s$ of $\mathfrak{S}$, that is, that $D$ is a derivation of $\mathfrak{S}$. It is sufficient to show that (22) holds for $r=b u, s=a+c u+z$ where $a$ and $c$ are in $\mathscr{B}$ and $u z=(1 / 2) z$. Then $(r s) D=[(b a+b c) u+(1 / 2) z b] D=[(b a+b c) D] u$ $+(1 / 2)(z b) D, \phi=(r D) s+r(s D)=[(b D) u] s+(b u)[a D+(c D) u+z D]$. We use the fact that $b D$ is in $\mathfrak{B}$ and $z D$ is in $y_{0} \mathfrak{B}+y_{1} \mathfrak{B}$ to obtain $\phi=(b D)[(a+c) u]$ $+(1 / 2) z(b D)+[b(a D)] u+[b(c D)] u+(1 / 2)(z D) b=[(b a+b c) D] u+(1 / 2)(z b) D$ as desired.

The relation $y_{0} y_{1}=0$ implies that $y_{0}\left(y_{1} D\right)+y_{1}\left(y_{0} D\right)=f_{0}^{\prime}-f_{1}^{\prime}=0$ and so

$$
f_{1}=f_{0}+\gamma,
$$

where $\gamma$ is in $\mathfrak{F}$.

Now $\quad\left(y_{0} x\right) D=y_{0}(x D)+y_{0} f_{0} x+\alpha y_{1} x \quad$ and $\quad y_{1}\left(y_{0} x\right)=1, \quad\left[y_{1}\left(y_{0} x\right)\right] D=0$ $=y_{1}\left[y_{0}(x D)+y_{0}\left(x f_{0}\right)\right]+\left(y_{0} x\right)\left(y_{1} f_{1}\right)=(x D)^{\prime}+\left(x f_{0}\right)^{\prime}+f_{1}-x f_{1}^{\prime}$ and so $(x D)^{\prime}+x f_{0}^{\prime}$ $+f_{0}+f_{0}+\gamma-x f_{0}^{\prime}=0$,

$$
(x D)^{\prime}+2 f_{0}+\gamma=0 .
$$

We are now ready to use (43), (42), and Lemma 20 to determine the derivations of $\mathfrak{T}$ and hence those of $\mathfrak{S}$. The first of these is given by the values $\gamma=x D=f_{0}=\beta=0$ and $\alpha=1$. This is the derivation $G$ where

$$
b G=\left(y_{1} b\right) G=0, \quad\left(y_{0} b\right) G=y_{1} b .
$$

To show that $G$ is actually a derivation of $\mathfrak{I}$ we see that (22) need only be verified for $r$ and $s$ in component subspaces $\mathfrak{B}, y_{0} \mathfrak{B}, y_{1} \mathfrak{B}$. One component must be in $y_{0} \&$ since otherwise all products are zero, $\left[\left(y_{0} b\right)\left(c+y_{0} c_{0}+y_{1} c_{1}\right)\right] G$ $=\left[y_{0}(b c)\right] G=y_{1}(b c),\left[\left(y_{0} b\right) G\right]\left[c+y_{0} c_{0}+y_{1} c_{1}\right]+\left(y_{0} b\right)\left[\left(y_{0} c_{0}\right) G\right]=y_{1}(b c)+\left(y_{1} b\right)\left(y_{0} c_{0}\right)$ $+\left(y_{0} b\right)\left(y_{1} c_{0}\right)=y_{1}(b c)$ as desired. Evidently the values $\gamma=x D=f_{0}=\alpha=0, \beta=1$ yields the transformation $H$ defined by

$$
b H=\left(y_{0} b\right) H=0, \quad\left(y_{1} b\right) H=y_{0} b,
$$

and symmetry considerations imply that $H$ is a derivation. But $b G H=b H G$ $=0,\left(y_{0} b\right) H G=0,\left(y_{0} b\right) G H=\left(y_{1} b\right) H=y_{0} b,\left(y_{1} b\right) H G=\left(y_{0} b\right) G=y_{1} b,\left(y_{1} b\right) G H=0$, and so $G H-H G=K$ where $K$ is the derivation defined by 


$$
b K=0, \quad\left(y_{0} b\right) K=y_{0} b, \quad\left(y_{1} b\right) K=-y_{1} b .
$$

Clearly $K$ corresponds to the values $x D=0=\alpha=\beta, \hat{f_{0}}=1, f_{1}=-1$.

It is easy to see that the derivations $G, H, K$ form a Lie algebra which is isomorphic to the set of all linear transformations of trace zero on the vector space $y_{0} \mathfrak{F}+y_{1} \mathfrak{F}$. The remaining derivations are those for which $\alpha=\beta=0$, $x D \neq 0, \gamma=0$. Then $f_{0}=-(1 / 2)(x D)^{\prime}$ and the general derivation of this kind is determined by

$$
x D_{c}=c, \quad y_{0} D_{c}=-(1 / 2) y_{0} c^{\prime}, \quad y_{1} D_{c}=-(1 / 2) y_{1} c^{\prime},
$$

where $c$ is any quantity of $\mathscr{B}$. Then for every $b$ of $\mathfrak{B}$ we have the definition

$$
b D_{c}=b^{\prime} c, \quad\left(y_{i} b\right) D_{c}=y_{i}\left(b^{\prime} c-(1 / 2) c^{\prime} b\right) \quad(i=0,1) .
$$

To verify that $D_{c}$ is a derivation of $\mathfrak{T}$ we first compute $\left[a\left(y_{i} b\right)\right] D_{c}$ $=\left[y_{i}(a b)\right] D_{c}=y_{i}\left[(a b)^{\prime} c-(1 / 2) c^{\prime}(a b)\right], \quad\left(a D_{c}\right)\left(y_{i} b\right)+a\left[\left(y_{i} b\right) D_{c}\right]=y_{i}\left(b a^{\prime} c\right)$ $+a\left[y_{i}\left(b^{\prime} c-(1 / 2) c^{\prime} b\right)\right]=y_{i}\left[(a b)^{\prime} c-(1 / 2) c^{\prime}(a b)\right]$ as desired. Since $D_{c}$ is known to induce a derivation in $\mathfrak{B}$, there is no need to consider (22) with $r$ and $s$ in $\mathfrak{B}$. It also holds trivially if $r$ and $s$ are in $y_{i} \mathfrak{B}$ since all terms are zero. There remains the case $r=y_{0} b, s=y_{1} a$ for $a$ and $b$ in $\mathfrak{B}$. Then $r s=b^{\prime} a-b a^{\prime}$, $(r s) D_{c}=\left(b^{\prime} a-b a^{\prime}\right)^{\prime} c, \quad\left(r D_{c}\right) s+r\left(s D_{c}\right)=\left[y_{0}\left(b^{\prime} c-(1 / 2) c^{\prime} b\right)\right]\left(y_{1} a\right)+\left(y_{0} b\right)\left[y_{1}\left(a^{\prime} c\right.\right.$ $\left.\left.-(1 / 2) c^{\prime} a\right)\right]=\left(b^{\prime} c-(1 / 2) c^{\prime} b\right)^{\prime} a-\left(b^{\prime} c-(1 / 2) c^{\prime} b\right) a^{\prime}+b^{\prime}\left(a^{\prime} c-(1 / 2) c^{\prime} a\right)-b\left(a^{\prime} c\right.$ $\left.\left.-(1 / 2) c^{\prime} a\right)^{\prime}=b^{\prime \prime} c+b^{\prime} c^{\prime}-(1 / 2) c^{\prime \prime} b-(1 / 2) c^{\prime} b^{\prime}\right) a-\left(b^{\prime} c-(1 / 2) c^{\prime} b\right) a^{\prime}+b^{\prime}\left(a^{\prime} c\right.$ $\left.-(1 / 2) c^{\prime} a\right)-b\left(a^{\prime \prime} c+a^{\prime} c^{\prime}-(1 / 2) c^{\prime \prime} a-(1 / 2) c^{\prime} a^{\prime}\right)=\left(b^{\prime \prime} a-b^{\prime} a^{\prime}+b^{\prime} a^{\prime}-b a^{\prime \prime}\right) c$ $+\left((1 / 2) b^{\prime} c^{\prime}-(1 / 2) c^{\prime \prime} b-(1 / 2) b^{\prime} c^{\prime}+(1 / 2) c^{\prime \prime} b\right) a+b\left((1 / 2) c^{\prime} a^{\prime}-(1 / 2) a^{\prime} c^{\prime}\right)$ $=\left(b^{\prime} a-b a^{\prime}\right)^{\prime} c$ as desired. This completes our verification that every $D_{c}$ is a derivation of $\mathfrak{T}$.

It is now easy to compute $D_{c} G-G D_{c}$. Indeed $b\left(D_{c} G-G D_{c}\right)=0$, $y_{0}\left(D_{c} G-G D_{c}\right)=-(1 / 2)\left(y_{0} c^{\prime}\right) G-y_{1} D_{c}=-(1 / 2) y_{1} c^{\prime}+(1 / 2) y_{1} c^{\prime}=0, \quad y_{1}\left(D_{c} G\right.$ $\left.-G D_{c}\right)=-(1 / 2)\left(y_{1} c^{\prime}\right) G-\left(y_{1} G\right) D_{c}=0$ and so $D_{c} G-G D_{c}=0$. It may similarly be shown that $D_{c} H-H D_{c}=0$. By the Jacobi identity $D_{c} K-K D_{c}=0$.

We have now obtained the structure of the algebra of all derivations of the form $\alpha_{0} G+\beta_{0} H+\gamma_{0} K+D_{c}$ for $\alpha_{0}, \beta_{0}, \gamma_{0}$ in $\mathfrak{F}$ and $c$ in $\mathfrak{B}$. It remains to show that this algebra is the algebra of all derivations $D$ of $\mathfrak{T}$. Let $D$ be any derivation and $y_{0} D=y_{0} f_{0}+\alpha y_{1}, y_{1} D=\beta y_{0}+y_{1} f_{0}+y_{1} \gamma$. Then $D^{\prime}=D-\alpha G$ $-\beta H+(1 / 2) \gamma K$ has the property that $y_{0} D^{\prime}=y_{0} f_{0}+\alpha y_{1}-\alpha y_{1}+(1 / 2) \gamma y_{0}$ $=y_{0}\left(f_{0}+(1 / 2) \gamma\right), \quad y_{1} D^{\prime}=y_{1} f_{0}+\beta y_{0}-\beta y_{0}+\gamma y_{1}-(1 / 2) \gamma y_{1}=y_{1}\left(f_{0}+(1 / 2) \gamma\right)$ and so we have a derivation $D^{\prime}$ of Lemma 20 with $\alpha=\beta=\gamma=0$. Let $x D=c$. Then $y_{0} D_{c}=-(1 / 2) y_{0} c^{\prime}, y_{1} D_{c}=-(1 / 2) y_{1} c^{\prime}$ and so $y_{i}\left(D^{\prime}-D_{c}\right)=y_{i}\left[f_{0}+(1 / 2)(x D)^{\prime}\right]$ $=0$ by $(43), x\left(D^{\prime}-D_{c}\right)=0$. Thus $D^{\prime}=D_{c}$ and we have proved the following result.

THEOREM 4. The derivation algebra of $\mathfrak{T}$ is the direct sum of the derivation algebra of $\mathfrak{B}$ and the three-dimensional simple Lie algebra.

5. A class of commutative power-associative algebras. Let $\mathfrak{B}$ be a com- 
mutative associative algebra with a unity quantity 1 and dimension $n$ over a field $\mathfrak{F}$ whose characteristic is prime to 30 . Suppose that $\mathfrak{T}$ is the vector space

$$
\mathfrak{T}=\mathfrak{B}+y_{0} \mathfrak{B}+\cdots+y_{m} \mathfrak{B},
$$

of dimension $n(m+2)$ over $\mathfrak{F}$, where $m>0$. Then we are assuming that a vector $b+y_{0} b_{0}+\cdots+y_{m} b_{m}=0$ for $b, b_{0}, \cdots, b_{m}$ in $\mathfrak{B}$ if and only if $b=b_{0}=\cdots=b_{m}=0$. Define

$$
\left(y_{i} b\right) c=c\left(y_{i} b\right)=y_{i}(b c)
$$

for every $b$ and $c$ of $\mathfrak{B}$. We also define

$$
\left(y_{i} b\right)\left(y_{j} c\right)=f_{i j}(b, c)=f_{j i}(c, b) \quad(i, j=0, \cdots, m),
$$

where the functions $f_{i j}(b, c)$ are bilinear functions of the variables $b$ and $c$ in $\mathfrak{B}$ with values in $\mathfrak{B}$. We have then defined a commutative algebra $\mathfrak{T}$, and we also assume that

$$
f_{i j}(1,1)=0 \quad(i, j=0, \cdots, m) .
$$

We shall now determine all functions $f_{i j}(b, c)$ such that the corresponding algebra $\mathfrak{T}$ is power-associative, that is, such that (5) holds for every $x, y, z, w$ of $\mathfrak{T}$.

We first substitute $x=a, y=y_{i} b, z=y_{j} c, w=y_{k} d$ in (5) where $a, b, c, d$ are in $\mathfrak{B}$, and we obtain

$$
y_{i} A_{i}+y_{j} A_{j}+y_{k} A_{k}=0,
$$

where we use the fact that $\mathfrak{B}$ is associative to derive the coefficient formulas

$$
\begin{aligned}
A_{i} & =\left[2 a f_{j k}(c, d)-f_{j k}(a c, d)-f_{j k}(c, a d)\right] b, \\
A_{j} & =\left[2 a f_{i k}(b, d)-f_{i k}(a b, d)-f_{i k}(b, a d)\right] c, \\
A_{k} & =\left[2 a f_{i j}(b, c)-f_{i j}(a b, c)-f_{i j}(b, a c)\right] d .
\end{aligned}
$$

Since $m>0$, we may select $i$ and $j$ to be distinct integers and take $k=j$. By our assumption about the dimension of $\mathfrak{I}$ we obtain $A_{i}=A_{j}+A_{k}=0$. Take $b=1$ in $A_{i}$ and $d=1$ in $A_{j}+A_{k}$ to obtain the identities

$$
\begin{aligned}
2 a f_{j j}(c, d) & -f_{j j}(a c, d)-f_{j j}(c, a d)=0, \\
{\left[2 a f_{i j}(b, 1)-f_{i j}(a b, 1)\right.} & \left.-f_{i j}(b, a)\right] c \\
+ & {\left[2 a f_{i j}(b, c)-f_{i j}(a b, c)-f_{i j}(b, a c)\right]=0 . }
\end{aligned}
$$

If we take $c=1$ in (58) and use the fact that the characteristic of $\mathfrak{F}$ is not two, we obtain

$$
2 a f_{i j}(b, 1)=f_{i j}(a b, 1)+f_{i j}(b, a) .
$$

But then (58) reduces to our fundamental relation 


$$
f_{i j}(b, a c)+f_{i j}(a b, c)=2 a f_{i j}(b, c),
$$

for $i \neq j$, and the relation also holds for $i=j$ by (57). Note that (59) is the case $c=1$ of $(60)$. If we also take $b=1$ and use the hypothesis that $f_{i j}(1,1)=0$, relation (59) yields

$$
f_{i j}(a, 1)+f_{i j}(1, a)=0 .
$$

Replace $b$ by $1, c$ by $a$, and $a$ by $b$ in (60). The result is

$$
f_{i j}(1, b a)+f_{i j}(b, a)=2 b f_{i j}(1, a) .
$$

Since $b a=a b$, we may use $(61)$ to write $f_{i j}(b, a)=f_{i j}(a b, 1)-2 b f_{i j}(a, 1)$ and we substitute this result in (59) to obtain

$$
f_{i j}(a b, 1)=a f_{i j}(b, 1)+b f_{i j}(a, 1) .
$$

But (63) implies that the linear transformation

$$
a \rightarrow f_{i j}(a, 1)=a D_{i j}
$$

is a derivation $D_{i j}$ of $\mathfrak{B}$. Since $f_{i j}(1, a)=-f_{i j}(a, 1)=-a D_{i j}=f_{j i}(a, 1)=a D_{j i}$, we have

$$
D_{i j}=-D_{j i}, \quad D_{i i}=0 \quad(i=0, \cdots, m) .
$$

Also $f_{i j}(a, b)=2 b\left(a D_{i j}\right)-(a b) D_{i j}=2 b\left(a D_{i j}\right)-\left[a\left(b D_{i j}\right)+\left(a D_{i j}\right) b\right]$ and so

$$
f_{i j}(a, b)=\left(a D_{i j}\right) b-a\left(b D_{i j}\right), \quad f_{i i}(a, b)=0 .
$$

Conversely (60) follows formally from (66).

We now assume (66) and its consequence (60) and propose to show that $\mathfrak{I}$ is power-associative. Let

$$
Y=\sum c_{i} y_{i}
$$

with $c_{i}$ in $\mathfrak{B}$. Then $(a Y)(b Y)=\sum_{i, j} f_{i j}\left(a c_{i}, b c_{j}\right)=(b Y)(a Y)=\sum_{i, j} f_{i j}\left(b c_{i}, a c_{j}\right)$ and so $Y^{2}=\sum_{i, j} f_{i j}\left(c_{i}, c_{j}\right)$. Since $Y^{2}$ is in the associative algebra $\mathfrak{B}$, we may use (69) to obtain $4 a\left(b Y^{2}\right)=\sum_{i, j} 2 a\left[f_{i j}\left(b c_{i}, c_{j}\right)+f_{i j}\left(c_{i}, b c_{j}\right)\right]=\sum_{i, j}\left[f_{i j}\left(a b c_{i}, c_{j}\right)\right.$ $\left.+f_{i j}\left(b c_{i}, a c_{j}\right)+f_{i j}\left(a c_{i}, b c_{j}\right)+f_{i j}\left(c_{i}, a b c_{j}\right)\right]$. But then (69) implies that $4 a\left(b Y^{2}\right)$ $=4(a b) Y^{2}=2(a b) Y^{2}+2(a Y)(b Y)$ and so

$$
(a Y)(b Y)=(a b) Y^{2}
$$

for every $a$ and $b$ of $\mathfrak{B}$. Since $Y^{2}$ is in $\mathfrak{B}$, it follows immediately that $\left(Y^{2} Y\right) Y$ $=Y^{2} Y^{2}$, and it is easy to see that $\left(t^{2} t\right) t=t^{2} t^{2}$ for every $t=a+Y$ of $\mathfrak{T}$. Thus $\mathfrak{T}$ is power associative $\left({ }^{7}\right)$ and we have completed our proof of the following result.

Theorem 5. Let $\mathfrak{I}=\mathfrak{B}+y_{0} \mathfrak{B}+\cdots+y_{m} \mathfrak{B}$ be the algebra defined by (49), tivity.

(7) The author is indebted to the referee for suggesting this simple proof of power associa- 
(50), (51), (52) where $\mathfrak{B}$ is a commutative associative algebra with unity quantity over a field $\mathfrak{F}$ of characteristic prime to 30. Then $\mathfrak{T}$ is power-associative if and only if the defining functions $f_{i j}(a, b)$ satisfy the relation $f_{i j}(a, b)=\left(a D_{i j}\right) b$ - $a\left(b D_{i j}\right)$ for derivations $D_{i j}=-D_{j i}$ of $\mathfrak{B}$.

The algebras $\mathfrak{T}$ depend only on $\mathfrak{B}$ and the set $\mathfrak{D}_{m}$ of the $(m+1)^{2}$ derivations $D_{i j}$ of $\mathfrak{B}$ and we shall write

$$
\mathfrak{T}=\mathfrak{T}\left(\mathfrak{B}, \mathfrak{D}_{m}\right) .
$$

Each algebra $\mathfrak{T}$ can be imbedded in an algebra

$$
\mathfrak{S}=\mathfrak{S}\left(\mathfrak{B}, \mathfrak{D}_{m}\right)=\mathfrak{T}\left(\mathfrak{B}, \mathfrak{D}_{m}\right)+u \mathfrak{B},
$$

such that $\mathfrak{S}$ contains an idempotent $u$ and is $u$-stable. We define $\mathfrak{S}$ by the relations

$$
u b=b u, \quad a(u b)=(a u) b=(a b) u
$$

for $a$ and $b$ in $\mathfrak{B}$, and by

$$
\left(y_{i} b\right)(u a)=(u a)\left(y_{i} b\right)=(1 / 2) y_{i}(a b) \quad(i=0,1, \cdots, m)
$$

for every $a$ and $b$ of $\mathfrak{B}$. If $\mathfrak{S}$ is power-associative, so is $\mathfrak{T}$. The converse follows readily by direct computation if we u'se (69) and the fact that $Y(b u)=(1 / 2) Y b$ for every $b$ of $\mathfrak{B}$ and $Y=\sum_{i} y_{i} c_{i}$.

TheOREM 6. The commutative algebra $\subseteq$ defined by (70), (71), (72) is powerassociative if and only if the corresponding subalgebra $\mathfrak{I}$ is power-associative.

6. On the simplicity of $\mathfrak{T}$. Let $\mathfrak{B}$ be any algebra and $\mathfrak{D}$ be the derivation algebra of $\mathfrak{B}, D$ be in $\mathfrak{D}$. Then an ideal $\mathfrak{M}$ of $\mathfrak{B}$ will be called a $D$-ideal if $\mathfrak{M}$ contains $c D$ for every $c$ of $\mathfrak{M}$. We call $\mathfrak{M}$ a $\mathfrak{D}$-ideal if $\mathfrak{M}$ is a $D$-ideal for every $D$ of $\mathfrak{D}$.

An algebra $\mathfrak{B}$ with a unity quantity will be called $D$-simple if $\mathfrak{B}$ and 0 are the only $D$-ideals of $\mathfrak{B}$. We say that $\mathfrak{B}$ is $\mathfrak{D}$-simple if $\mathscr{B}$ and 0 are the only $\mathfrak{D}$-ideals of $\mathfrak{B}$.

Let us now consider the algebra $\mathfrak{T}$ of $\$ 5$ where $\mathfrak{B}$ is an associative algebra with a unity quantity. We shall prove:

TheOREM 7. The algebra $\mathfrak{T}$ is simple only if $\mathfrak{B}$ is $\mathfrak{D}$-simple.

For let $\mathfrak{B}$ contain a $\mathfrak{D}$-ideal $\mathfrak{M}$. Then $\mathfrak{S}=\mathfrak{M}+\sum_{i=0}^{m} y_{i} \mathfrak{M}$ has the property that $\mathfrak{H B O} \subseteq$ S. Also $\left(y_{i} \mathfrak{M}\right) \mathfrak{B}=y_{i}(\mathfrak{M P}) \subseteq y_{i} M$ and $\left(y_{i} \mathfrak{M}\right)\left(y_{i} \mathfrak{B}\right) \subseteq \mathfrak{M}$ since $\left(y_{i} b\right)\left(y_{i} c\right)=\left(b D_{i j}\right) c-b\left(c D_{i j}\right)$ is in $\mathfrak{M}$ for every $b$ of $\mathfrak{M}$ and $c$ of $\mathfrak{B}$. Thus $\mathbb{B}$ is an ideal of $\mathfrak{T}$. But $\mathfrak{S} \neq \mathfrak{T}$ since $\mathfrak{M}$ is a proper ideal of $\mathfrak{B}$.

THEOREM 8. Let $\mathfrak{B}$ be a commutative associative algebra with a unity quantity over $\mathfrak{F}$ and $\mathfrak{B}$ be $\mathfrak{D}$-simple. Then $\mathfrak{F}$ has characteristic $p, \mathfrak{B}=\mathfrak{F}+\mathfrak{N}$ where $\mathfrak{N}$ is the radical of $\mathfrak{B}, x^{p}=0$ for every $x$ of $\mathfrak{R}$. 
For if $\mathfrak{B}$ contained an idempotent $u \neq 1$ it would be true that $\mathfrak{B}=\mathfrak{B} u$ $\oplus \mathfrak{B}(1-u)$. Now $u^{2}=u, 2 u(u D)=u D,[2 u(u D)] u=2 u(u D)=u(u D), u(u D)$ $=u D=0,(b u) D=(b D) u$ is in $\mathfrak{B} u$ for every $D$ of $\mathfrak{D}$ and $b$ of $\mathfrak{B}$. But then $\mathfrak{B} u \neq \mathfrak{B}$ is a $\mathfrak{D}$-ideal of $\mathfrak{B}$ contrary to hypothesis. Hence $\mathfrak{B}=\mathfrak{F}+\mathfrak{N}$. If $x$ is any element of $\mathfrak{N}$ and $x^{t}=0, x^{t-1} \neq 0$, then $t x^{t-1}(x D)=0$. If $\mathfrak{F}$ has characteristic zero, then $x^{t-1}(x D)=0, x D$ is singular for every $x$ of $\mathfrak{N}$ and $D$ of $\mathfrak{D}$, $x D$ is in $\mathfrak{R}$ for every $x$ of $\mathfrak{R}, \mathfrak{N}$ is a $\mathfrak{D}$-ideal of $\mathfrak{B}$. Hence $\mathfrak{B}$ must have characteristic $p$. Let $\mathfrak{M}$ be the set of all $x^{p}$ for $x$ in $\mathfrak{N}, \mathbb{S}=\mathfrak{M} \mathscr{B}$. Clearly $\mathbb{B}$ is a proper ideal of $\mathfrak{B}$. Also $B$ is a $\mathfrak{D}$-ideal since $\left(x^{p} b\right) D=x^{p}(b D)+p\left[x^{p-1}(x D)\right] b=x^{p}(b D)$ is in $\mathbb{B}$ for every $x$ of $\mathfrak{N}$ and $b$ of $\mathfrak{B}$. Thus $\mathbb{B}=0, x^{p}=0$ for every $x$ of $\mathfrak{N}$.

It is not known whether there exist any $\mathfrak{D}$-simple algebras other than the class we shall consider in the next section.

7. The algebra $\mathfrak{B}_{r}$. Let $\mathfrak{F}$ be a field of characteristic $p>5$ and

$$
\mathfrak{B}=\mathfrak{B}_{r}=\mathfrak{F}\left[x_{1}, \cdots, x_{r}\right]
$$

be the commutative associative algebra of all polynomials

$$
b=b\left(x_{1}, \cdots, x_{r}\right)=\sum_{i_{n}=0}^{p-1} \beta_{i_{1} \ldots i_{n}} x_{1}^{i_{1}} \cdots x_{r}^{i_{r}},
$$

with coefficients $\beta_{i_{1} \ldots i_{n}}$ in $\mathfrak{F}$ such that $x_{\mathbf{k}}^{0}=1, x_{\mathbf{k}}^{p}=0$. We assume that $\mathfrak{B}$ has dimension $n=p^{r}$ over $\mathfrak{F}$, that is, that $b=0$ if and only if $\beta_{i_{1} \cdots i_{r}}=0$. We shall prove that $\mathfrak{B}$ is $\mathfrak{D}$-simple and shall actually prove the stronger result which states that there exists a derivation $D$ of $\mathscr{B}$ such that $\mathscr{B}$ is $D$-simple.

Consider the set $\&$ of all nonzero power products

$$
g=g\left[\alpha_{1}, \cdots, \alpha_{r}\right]=x_{1}^{\alpha_{1}} \cdots x_{r}^{\alpha_{r}} \quad\left(0 \leqq \alpha_{p}<p\right) .
$$

The set $\&$ contains precisely $p^{r}$ elements and we may order (B) by writing $g\left[\alpha_{1}, \cdots, \alpha_{r}\right]<g\left[\beta_{1}, \cdots, \beta_{r}\right]$ if $\alpha_{k+i}=\beta_{k+i}$ for $i=1,2, \cdots, r-k$ but $\alpha_{k}$ $<\beta_{k}$. Then the least element of $B$ is $1=g[0, \cdots, 0]$. The greatest element of (S) is

$$
z=z_{r}=g[p-1, \cdots, p-1]=\left(x_{1} \cdots x_{r}\right)^{p-1} .
$$

Lemma 21. Let $b \neq 0$ be in $\mathfrak{B}_{r}$. Then there exists an element $g$ of $\&$ such that $b g=\gamma z_{r}$ where $\gamma \neq 0$ is in $\mathfrak{F}$.

For $g z_{r}=0$ for every $g>1$ in $B$. If the constant term of $b$ is $\gamma \neq 0$, it should be clear that $b z_{r}=\gamma z_{r}$. Otherwise $b z_{r}=0, b 1 \neq 0$, and there must exist an element $g$ in \&s such that $c=b g \neq 0, b h_{i}=0$ for every $h_{i}=g x_{i}$. Then $c x_{i}=0$ for $i=1, \cdots, r$ and (30) implies that $c=\gamma z_{r}$ for $\gamma \neq 0$ in $\mathfrak{F}$.

Nathan Jacobson has shown $\left(^{8}\right)$ that the derivation algebra of $\mathfrak{B}_{r}$ is the set of all linear transformations $b \rightarrow b D\left(a_{1}, \cdots, a_{n}\right)$ of $\mathfrak{B}_{r}$ where

(8) Classes of restricted Lie algebras of characteristic p. II, Duke Math. J. vol. 10 (1943) pp. 107-121. 


$$
b D\left(a_{1}, \cdots, a_{r}\right)=\frac{\partial b}{\partial x_{1}} a_{1}+\cdots+\frac{\partial b}{\partial x_{r}} a_{r}
$$

for $a_{1}, \cdots, a_{r}$ in $\mathfrak{B}_{r}$, and the symbol $\partial b / \partial x_{i}$ is the ordinary partial derivative. We shall actually show that $\mathfrak{B}_{r}$ is $W$-simple where

$$
W=D\left(z_{0}, \cdots, z_{r-1}\right)
$$

and $z_{0}=1$. We now prove:

LEммA 22. Let $g \neq 1$ be in (5. Then $g W=\alpha h$ where $\alpha$ is an integer, $h$ is in (S) and $h<g$.

For let $g=g\left[\alpha_{1}, \cdots, \alpha_{r}\right]$ such that $\alpha_{1}=\cdots=\alpha_{8-1}=0$ and $\alpha_{8} \neq 0$. Then $g=x_{s}^{\alpha} q$ where $\alpha=\alpha_{8}>0$ and $q=g\left[0, \cdots, 0, \alpha_{s+1}, \cdots, \alpha_{r}\right], g W=\alpha x_{s}^{\alpha-1} z_{s-1} q$ $+x_{s}^{\alpha}(q W)$. Since $q W$ has $z_{s}$ as a factor and $x_{s} z_{s}=0$, we see that $g W=\alpha x_{s}^{\alpha-1} z_{s-1} q$ $=\alpha h$ where $h=g\left[p-1, \cdots, p-1, \alpha-1, \alpha_{s+1}, \cdots, \alpha_{r}\right]<g$ as desired.

Since $B$ is a finite set and $g>g W \alpha^{-1}>g W^{2} \beta^{-1}>\cdots$, we may combine this result with Lemma 21 and have:

LEMMA 23. Let $b$ be any nonzero quantity of $\mathfrak{B}_{r}$. Then there exists a quantity $g$ in $\mathfrak{B}$ and an integer $k$ such that $b g=z, z W^{k}=1$. Thus $\mathfrak{B}$ is $W$-simple.

8. New central simple algebras. Let $\mathfrak{B}=\mathfrak{B}_{r}=\mathfrak{F}\left[1, x_{1}, \cdots, x_{r}\right]$ be the commutative associative polynomial algebra of dimension $p^{r}$ over $\mathfrak{F}$ of characteristic $p$ where $x_{i}^{p}=0$. The definition of a set $\mathfrak{D}_{m}$ of derivations $D_{i j}$ results in a corresponding algebra

$$
\mathfrak{T}=\mathfrak{T}\left(\mathfrak{B}, \mathfrak{D}_{m}\right)
$$

of dimension $p^{r}$ over $\mathfrak{F}$. The subspace

$$
\mathfrak{R}=\mathfrak{R}\left(\mathfrak{B} ; \mathfrak{M}_{0}, \cdots, \mathfrak{M}_{m}\right)=\mathfrak{B}+y_{0} \mathfrak{M}_{0}+\cdots+y_{m} \mathfrak{M}_{m}
$$

is a subalgebra of $\mathfrak{T}\left(\mathfrak{B}, \mathfrak{D}_{m}\right)$ for all ideals $\mathfrak{M}_{0}, \cdots, \mathfrak{M}_{m}$ of $\mathfrak{B}$. Moreover, if

$$
\mathfrak{M}_{0}=\mathfrak{M}_{1}=\cdots=\mathfrak{M}_{s}=\mathfrak{B}, \quad \mathfrak{M}_{s+1}=\cdots=\mathfrak{M}_{m}=0
$$

then

$$
\mathfrak{R}\left(\mathfrak{B} ; \mathfrak{M}_{0}, \cdots, \mathfrak{M}_{m}\right)=\mathfrak{T}\left(\mathfrak{B}, \mathfrak{D}_{s}\right),
$$

where $\mathfrak{D}_{8}$ is obtained from $\mathfrak{D}_{m}$ by deleting all derivations $D_{i j}$ with either $i>s$ or $j>s$.

We shall define a class of central simple algebras $\mathfrak{T}\left(\mathfrak{B}, \mathfrak{D}_{m}\right)$ by defining a set $\leftleftarrows$ of derivations $D_{i j}$ for all integers $j>i \geqq 0$. The sets $\mathfrak{D}_{m}$ will then be obtained by deleting those derivations $D_{i j}$ with $i>m$ or $j>m$. We first define the derivations which are actually in the set $\&$ by

$$
D_{1}=W, \quad b G=\frac{\partial b}{\partial x_{r}} x_{r}, \quad b D_{k}=\frac{\partial b}{\partial x_{k}} \quad(k=2, \cdots, r) .
$$


We then assume that $D_{i j}=0$ except when $i=t r, j=t r+k$ for all integers $t \geqq 0$ and $k=1, \cdots, r$. For the latter values of $i$ and $j$ we define

$$
D_{t r, t r+k}=D_{k} \quad(t=0,2, \cdots ; k=1, \cdots, r),
$$

and

$$
D_{r, r+k}=D_{k}, \quad D_{r, 2 r}=G \quad(k=1, \cdots, r-1) .
$$

This completes our definition of the set $\&$ and the corresponding algebras $\mathfrak{T}\left(\mathfrak{B}, \mathfrak{D}_{m}\right)$.

We shall show that the algebras $\mathfrak{T}\left(\mathfrak{B}, \mathfrak{D}_{m}\right)$ are central simple over $\mathfrak{F}$. Indeed this is the special case with $\mathfrak{M}_{i}=\mathfrak{B}$ of the following result.

THEOREM 9. Let $\mathfrak{R}=\mathfrak{R}\left(\mathfrak{B} ; \mathfrak{M}_{0}, \cdots, \mathfrak{M}_{m}\right)$ where the $\mathfrak{M}_{i}$ are ideals of $\mathfrak{B}=\mathfrak{B}$ r such that $\mathfrak{M}_{1}=\mathfrak{M}_{\text {tr }}=\mathfrak{B}$ for all $t$ in the set defined by $0 \leqq t r<m$. Then $\mathfrak{R}$ is a central simple algebra.

We shall use the fact that $\mathfrak{R}$ may be regarded as a subalgebra of $\mathfrak{T}\left(\mathfrak{B}, \mathfrak{D}_{n}\right)$ where

$$
r(s-1)<m \leqq n=r s, \quad s>0 .
$$

Then $\mathfrak{Q}$ contains $y_{t r}$ for $t=0, \cdots, s-1$. The general element of $\mathfrak{R}$ now has the form

$$
h=b+y_{0} b_{0}+\cdots+y_{m} b_{m}
$$

for $b, b_{0}, \cdots, b_{m}$ in $\mathfrak{B}$ where actually the $b_{i}$ are in $\mathfrak{M}_{i}$. We shall require the following preliminary result.

Lemma 24. Let $d=\left(\alpha_{0} y_{0}+\cdots+\alpha_{m} y_{m}\right) z$ for $\alpha_{0}, \cdots, \alpha_{m}$ in $\mathfrak{F}$ where $z$ $=\left(x_{1} \cdots x_{r}\right)^{p-1}$. Then $y_{t r} d=0$ for $t=0, \cdots, s-1$ if and only if $d=0$.

The definition (78) means that

$$
\left(y_{t r} b\right) y_{t r+k}=-y_{t r}\left(y_{t r+k} b\right)=b D_{k}(t=0,2, \cdots, s-1 ; k=1, \cdots, r),
$$

and (79) means that

$$
\begin{array}{r}
\left(y_{r} b\right) y_{r+k}=-y_{r}\left(y_{r+k} b\right)=b D_{k}, \quad\left(y_{r} b\right) y_{2 r}=-y_{r}\left(y_{2 r} b\right)=b G \\
(k=1, \cdots, r-1) .
\end{array}
$$

If $y_{0} d=y_{0}\left[\left(y_{0} \alpha_{0}+\cdots+y_{r} \alpha_{r}\right) z\right]=-\left(\left(\partial z / \partial x_{1}\right) \alpha_{1}+\left(\partial z / \partial x_{2}\right) \alpha_{2}+\cdots+\left(\partial z / \partial x_{r}\right) \alpha_{r}\right)$ $=0$, then $\alpha_{1}=\cdots=\alpha_{r}=0$ since the $r$ partial derivatives $\partial z / \partial x_{i}$ are clearly linearly independent monomials. We next form $y_{r} d=\left[\left(y_{0} \alpha_{0}+y_{r+1} \alpha_{r+1}+\cdots\right.\right.$ $\left.\left.+y_{2 r} \alpha_{2 r}\right) z\right] y_{r}=\left(\partial z / \partial x_{r}\right) \alpha_{0}-\left(\left(\partial z / \partial x_{1}\right) \alpha_{r+1}+\cdots+\left(\partial z / \partial x_{r-1}\right) \alpha_{2 r-1}\right)-\alpha_{2 r} x_{r} \partial z / \partial x_{r}$. Since $-x_{r} \partial z / \partial x_{r}=z$, we see that $y_{r} d=0$ if and only if $\alpha_{0}=\alpha_{r+1}=\cdots=\alpha_{2 r}$ $=0$. If $\alpha_{0}=\cdots=\alpha_{t r}=0$, then $y_{r t} d=y_{r t}\left[\left(y_{t r+1} \alpha_{t r+1}+\cdots+y_{r(t+1)} \alpha_{r(t+1)}\right) z\right]$ $=-\left[\left(\partial z / \partial x_{1}\right) \alpha_{r t+1}+\cdots+\left(\partial z / \partial x_{r}\right) \alpha_{r(t+1)}\right]=0$ if and only if $\alpha_{r t+1}=\cdots$ $=\alpha_{r(t+1)}=0$. This completes our proof of the lemma. 
Assume that $\mathfrak{W}$ is a nonzero ideal of $\mathfrak{R}$ and that $\mathfrak{S}_{0}$ is the intersection of $\mathfrak{S}$ and $\mathfrak{B}$. If $\mathfrak{S}_{0} \neq 0$, we use Lemma 21 to see that $z$ is in $\mathfrak{S}_{0}$. But then $\left(y_{0} z\right) y_{1}$ $=z W$ is in $\mathfrak{S}_{0}$ and a trivial induction implies that $\left(y_{0} z W^{k-1}\right) y_{1}=z W^{k}$ is in $\mathfrak{S}_{0}$. By Lemma 23 we see that $\mathfrak{S}_{0}$ contains the unity quantity of $\mathfrak{B}$ and so $\mathfrak{S}$ contains $\mathfrak{R}, \mathfrak{S}=\mathfrak{R}$.

Assume now that $\mathfrak{S}_{0}=0$ whence $\mathfrak{S} \neq \mathfrak{R}$. If $h$ is a nonzero quantity of $\mathfrak{S}$, we use (81) and form $y_{0} h=y_{0} b+c$ where $c=y_{0}\left(y_{0} b_{0}+\cdots+y_{m} b_{m}\right)$ is in $\mathfrak{B}$. If $c \neq 0$, then $y_{0}\left(y_{0} h\right)=y_{0} c$ is in $\mathfrak{S},\left(y_{0} c\right) y_{1}=c W$ is in $\mathfrak{S}_{0}$ and must be zero, $\left(y_{1} x_{1}\right)\left(y_{0} c\left(=(c W) x_{1}-c=-c\right.\right.$ is in $\mathfrak{S}_{0}$, a contradiction. Hence $c=0, y_{0} h=y_{0} b$. Then $y_{1}\left(y_{0} b\right)=b W$ is in $\mathfrak{S}_{0}$ and is zero, $\left(y_{1} x_{1}\right)\left(y_{0} b\right)=-b$ is in $\mathfrak{S}_{0}, b=0, h=y_{0} b_{0}$ $+\cdots+y_{m} b_{m}$ and so $\mathfrak{S} \subseteq y_{0} \mathfrak{M}_{0}+\cdots+y_{m} \mathfrak{M}_{m}$. But $y_{t r}$ is in $\mathfrak{R}$ for $t=0,1, \cdots$, $s-1$ and $y_{t r} h$ is in $\mathfrak{S}_{0}$ for $t=0, \cdots, s-1$. Thus $y_{t r} h=0$ and Lemma 24 implies that $h=0$ contrary to hypothesis. This proves that $\&$ is a simple algebra. Since the proof remains true under scalar extension, $\mathbb{R}$ is central simple.

Each of the algebras $\mathfrak{R}=\mathfrak{R}\left(\mathfrak{B} ; \mathfrak{M}_{0}, \cdots, \mathfrak{M}_{m}\right)$ is a subalgebra of a $u$-stable algebra

$$
\mathfrak{Q}=\mathfrak{Q}\left(\mathfrak{B} ; \mathfrak{M}_{0}, \cdots, \mathfrak{M}_{m}\right)=\mathfrak{l}+u \mathfrak{B}
$$

which is defined by the multiplication table of $\mathbb{R}$ and the relation

$$
\left(y_{i} b\right)(c u)=(c u)\left(y_{i} b\right)=(1 / 2) y_{i}(b c)
$$

for every $b$ of $\mathfrak{M}_{i}$ and $c$ of $\mathfrak{B}$.

THEOREM 10. If $\mathbb{R}$ is central simple, so is the corresponding algebra $\mathfrak{Q}$.

For let $\mathfrak{W}$ be a nonzero ideal of $\mathfrak{Q}$ and $\mathfrak{S}^{\prime}$ be the intersection of $\mathfrak{E}$ and $\mathfrak{R}$. Then $\mathfrak{S}^{\prime}$ is an ideal of $\mathfrak{R}$ and if $\mathfrak{Z}$ is central simple, it follows that $\mathfrak{S}^{\prime}=\mathfrak{R}, \mathfrak{Y}=\mathfrak{Q}$ or that $\mathfrak{S}^{\prime}=0$. Let $\mathfrak{S}^{\prime}=0$ and $\mathfrak{S} \neq 0$. Then there exists an element $h=b+c u$ $+y_{0} b_{0}+\cdots+y_{m} b_{m} \neq 0$ in $\mathfrak{S}$ where $b$ and $c$ are in $\mathfrak{B}$ and $b_{i}$ is in $\mathfrak{M}_{i}$. Let $v=1-u$ so that $v h=b v+(1 / 2)\left(y_{0} b_{0}+\cdots+y_{m} b_{m}\right)$ is in $\mathfrak{S}, y_{0}(v h)=(1 / 2) y_{0} b+d$ is in $\mathfrak{S}^{\prime}$ where $d$ is in $\mathfrak{B}$. Then $b=d=0, h y_{0}=(1 / 2) y_{0} c+e$ for $e$ in $\mathfrak{B}, c=0, h$ is in $\mathfrak{S}^{\prime}$ contrary to hypothesis. This completes our proof.

It would be interesting to study the question of isomorphism for the various algebras which may be constructed by the methods we have presented here. In particular it would be desirable to know whether the algebras $\mathfrak{R}$ contain idempotents $u$ such that $\mathbb{R}$ is $u$-stable. We leave these questions for later study.

The University of Chicago,

Chicago, Ill. 